\title{
On the Energy-Momentum Spectrum of a Homogeneous Fermi Gas
}

\author{
Jan Dereziński, Krzysztof A. Meissner and Marcin Napiórkowski
}

\begin{abstract}
We consider translation-invariant quantum systems in thermodynamic limit. We argue that their energy-momentum spectra should have shapes consistent with effective models involving quasiparticles. Our main example is second quantized homogeneous interacting Fermi gas in a large cubic box with periodic boundary conditions, at zero temperature. We expect that its energy-momentum spectrum has a positive energy gap and a positive critical velocity.
\end{abstract}

\section{Introduction}

\subsection{Excitation Spectrum of Fermi gas}

In [8], one of the authors of this paper together with H. Cornean and P. Ziń discussed a number of conjectures about the excitation spectrum of the interacting Bose gas at zero temperature with repulsive potentials in the thermodynamic limit. In particular, [8] conjectured that such systems have a quasiparticle-like excitation spectrum without an energy gap and with a positive critical velocity. These conjectures seem to be consistent with experimental data. In particular, they explain various phenomena related to the superfluidity.

In this paper, we would like to sketch a number of analogous conjectures about the interacting Fermi gas at zero temperature with an attractive interaction in thermodynamic limit. We will argue that these systems should possess a quasiparticle-like excitation spectrum with a positive energy gap and a positive critical velocity. This conjecture implies in particular that the ground state energy is separated from the rest of the spectrum. In some situations, this ground state can be interpreted as a current carrying state, and plays an important role in the phenomenon of superconductivity.

The most robust quantity related to the excitation spectrum seems to be its infimum. Therefore, our main conjectures involve the infimum of the excitation spectrum (separately in the even and odd sector). They are based on the grand-canonical Hamiltonian for a fixed chemical potential. 
However, to have full information about the excitation spectrum, it is not enough to know its infimum. In fact, the HFB approximation suggests that the excitation spectrum of the Fermi gas has "lacunas" near its bottom. We make an attempt to express some conjectures about these lacunas. These conjectures are more complicated to state, probably also more delicate, and involve the canonical Hamiltonian, (that is, for a fixed number of particles).

We do not prove our conjectures. However, we introduce a rather general class of model Hamiltonians for which these conjectures can be tested and, maybe, proven under some assumptions. These Hamiltonians consist of a two-body kinetic energy, not necessarily quadratic, and an interaction. The interaction does not have to be local (given by a local potential). We also suppose that our system has "internal degrees of freedom" (e.g., "spin"). It seems important to assume that the interaction is in some sense "attractive", which means some kind of negative definiteness. This is suggested by the HartreeFock-Bogoliubov (HFB) method.

We include in our paper short computations based on the HFB approach. The basic principle of these computations is well known, but in the literature they are usually presented in simple special cases. Our presentation applies to a rather general case. As a result of the HFB approach we obtain an approximate quasiparticle representation of our Hamiltonian with a dispersion relation possessing very special features: a positive energy gap and a positive critical velocity. We conjecture that this dispersion relation suggests basic qualitative features of the true excitation spectrum of interacting Fermi gas in thermodynamic limit.

\subsection{Role of Translation Invariance}

There exist many papers that study the energy spectrum of interacting Fermi and Bose systems. In particular, there are interesting works that study the HBF approximation in such systems. What makes our paper different is the role of translation invariance. This enables us to ask questions about the excitation spectrum, which we expect to have interesting properties.

Many papers attempt to show that models based on quasiparticles give some kind of an approximation to realistic Hamiltonians, see e.g., [5, 9]. However, only relatively crude features are considered in essentially all these papers. Typically, they study the energy or the free energy per volume in thermodynamic limit. We are interested in the excitation spectrum, which is a finer quantity and does not involve dividing by the large volume. The only rigorous result that we know devoted to the excitation spectrum of an interacting quantum gas is due to Seiringer [21]. It concerns the Bose gas in finite volume and a mean field limit.

We always assume that the interaction is translation invariant. In realistic physical systems translation invariance is at most approximate. Superconducting materials are perhaps the closest to idealized translation-invariant models that we consider. Nevertheless, we believe that the picture presented in our paper is physically relevant also in many Fermi systems that are quite far from 
being translationally invariant, such as quantum dots and nuclei. In fact, one can argue that "traces" of translational degrees of freedom are present also in these systems, disguised as rotations and vibrations. In particular, the energy spectra of their odd and even sectors have various features consistent with our conjectures, see [3] for quantum dots and [19, Sect. 6.1] for nuclei.

The immediate motivation of our paper is to state mathematically interesting rigorous conjectures together with heuristic arguments in their favor. Therefore, we do not strive at all costs to describe realistic concrete physical systems. The assumption of translation invariance helps to formulate a clean and rigorous definition of various concepts.

\section{Quasiparticles and Quasiparticle-Like Excitation Spectrum}

The concept of "quasiparticle", although often used, seems to have no satisfactory definition in the literature. In this section we attempt to give a number of rigorous interpretations of this term. We will also discuss spectral properties of quantum systems that can be described in terms of quasiparticles.

The discussion of this section will be rather general and abstract. To a large extent it will be independent of the rest of the paper.

\subsection{Translation-Invariant Quantum Systems}

The main object of interest of this paper are translation-invariant quantum systems in thermodynamic limit. There are at least two approaches that can be used to describe such systems.

In the first approach one starts with a construction of a system in finite volume, using $\Lambda=[-L / 2, L / 2]^{d}$, the $d$-dimensional cubic box of side length $L$, as the configuration space. It is convenient, although somewhat unphysical, to impose the periodic boundary conditions, The system is described by its Hilbert space $\mathcal{H}^{L}$, Hamiltonian $H^{L}$ and momentum $P^{L}$. The spectrum of the momentum is discrete and coincides with $\frac{2 \pi}{L} \mathbb{Z}^{d}$. After computing appropriate quantities (such as the infimum of the excitation spectrum) one tries to take the $\operatorname{limit} L \rightarrow \infty$.

Sometimes a different approach is possible. One can try to construct a Hilbert space $\mathcal{H}$, a Hamiltonian $H$ and a momentum $P$ that describe the system on $\mathbb{R}^{d}$. This may be not easy. It may require the use of refined techniques $[7,16]$. It is probably not always possible. Note that in this case the spectrum of the momentum is expected to be absolutely continuous, with the exception of the ground state.

The latter approach seems conceptually more elegant. Throughout most of this section we will adopt it. In most situations this will allow us to formulate some of the physical concepts in a concise manner. (Sometimes, however, it will lead to technical complications).

In the next two sections we adopt the former approach, which is more down-to-earth. Thus only a family $\left(H^{L}, P^{L}\right)$ for finite $L$ will be defined.

To sum up, throughout most of this section by a translation-invariant quantum system we will mean $d+1$ commuting self-adjoint operators 
$\left(H, P_{1}, \ldots, P_{d}\right)$ on a Hilbert space $\mathcal{H}$. $H$ has the interpretation of a Hamiltonian and $P=\left(P_{1}, \ldots, P_{d}\right)$ describes the momentum.

\subsection{Excitation Spectrum}

The joint spectrum of the operators $(H, P)$ (which is a subset of $\mathbb{R}^{1+d}$ ) will be denoted by $\operatorname{sp}(H, P)$ and called the energy-momentum spectrum of $(H, P)$.

We will often assume that $H$ is bounded from below. If it is the case, we can define the ground state energy as $E:=\inf \operatorname{sp} H$. We will also often assume that $H$ possesses translation-invariant ground state $\Phi$, which is a unique joint eigenvector of $H, P$. In particular, $H \Phi=E \Phi$ and $P \Phi=0$.

Under these assumptions, by subtracting the ground state energy from the energy-momentum spectrum we obtain the excitation spectrum of $(H, P)$, that is, $\operatorname{sp}(H-E, P)$. We can also introduce the strict excitation spectrum as the joint spectrum of restriction of $(H-E, P)$ to the orthogonal complement of $\Phi$ :

$$
\operatorname{Exc}:=\operatorname{sp}\left(\left.(H-E, P)\right|_{\{\Phi\}^{\perp}}\right)
$$

Thus if $(E, \mathbf{0})$ is an isolated simple eigenvalue of $(H, P)$, then

$$
\mathrm{Exc}=\operatorname{sp}(H-E, P) \backslash(0, \mathbf{0}) .
$$

Otherwise $\operatorname{Exc}=\operatorname{sp}(H-E, P)$.

We introduce also a special notation for the infimum of Exc:

$$
\epsilon(\mathbf{k}):=\inf \{e:(e, \mathbf{k}) \in \operatorname{Exc}\} .
$$

The following two parameters have interesting physical implications. The first is the energy gap, defined as

$$
\varepsilon:=\inf \left(\left.\operatorname{sp}(H-E)\right|_{\{\Phi\}^{\perp}}\right)=\inf \left\{\epsilon(\mathbf{k}): \mathbf{k} \in \mathbb{R}^{d}\right\} .
$$

Another quantity of physical interest is the critical velocity:

$$
c_{\mathrm{cr}}:=\inf _{\mathbf{k} \neq \mathbf{0}} \frac{\epsilon(\mathbf{k})}{|\mathbf{k}|} .
$$

Physical properties of a system are especially interesting if the energy gap $\varepsilon$ is strictly positive. In such a case, the ground state energy is separated from the rest of the energy spectrum, and hence the ground state is stable.

Positive critical velocity is also very interesting. Physically, a positive critical velocity is closely related to the phenomenon of superfluidity, see e.g., a discussion in [8].

\subsection{Essential Excitation Spectrum}

One expects that most of a typical excitation spectrum is absolutely continuous wrt. the Lebesgue measure on $\mathbb{R}^{d+1}$. However, it may also contain isolated shells continuously depending on the momentum. In this subsection we attempt to define the part of the excitation spectrum that corresponds to such a situation. 
Note that this is not easy in the abstract framework that we adopted in this section. Actually, in the next section, based on finite volume systems $\left(H^{L}, P^{L}\right)$, we will use a different approach to define isolated shells, see Sect. 3.7.

We say that $(e, \mathbf{k}) \in \mathbb{R}^{d+1}$ belongs to $\mathrm{Exc}_{\mathrm{d}}$, called the discrete excitation spectrum, if there exists $\delta>0$ such that the operator $P$ has an absolutely continuous spectrum of uniformly finite multiplicity when restricted to

$$
\operatorname{Ran} \mathbb{1}(|H-E-e|<\delta) \mathbb{1}(|P-\mathbf{k}|<\delta) .
$$

The essential excitation spectrum is defined as $\operatorname{Exc}_{\mathrm{ess}}:=\operatorname{Exc} \backslash \mathrm{Exc}_{\mathrm{d}}$.

(We use an obvious notation for spectral projections of self-adjoint operators $H$ and $P$ : e.g., $\mathbb{1}(|H-e|<\delta)$ denotes the spectral projection of $H$ onto ]$e-\delta, e+\delta[$.

We introduce also a special notation for the bottom of Exc $_{\mathrm{ess}}$ :

$$
\epsilon_{\mathrm{ess}}(\mathbf{k}):=\inf \left\{e:(e, \mathbf{k}) \in \operatorname{Exc}_{\mathrm{ess}}\right\} .
$$

Obviously,

$$
\begin{gathered}
\operatorname{Exc} \supset \operatorname{Exc}_{\mathrm{ess}}, \\
\epsilon(\mathbf{k}) \leq \epsilon_{\mathrm{ess}}(\mathbf{k}), \quad \mathbf{k} \in \mathbb{R}^{d} .
\end{gathered}
$$

Note that typically $\operatorname{Exc}_{\mathrm{d}}$ consists of a finite number of shells separated by lacunas.

Abstract theory allows us to represent the Hilbert space $\mathcal{H}$ as the direct integral over $\mathbb{R}^{d}$ given by the spectral decomposition of $P$, see e.g., [6], 4.4.1. Suppose, in addition, that this direct integral can be taken with respect to the Lebesgue measure, so that we can write

$$
H=\int_{\mathbf{k} \in \mathbb{R}^{d}}^{\oplus} H(\mathbf{k}) \mathrm{d} \mathbf{k} .
$$

Then it is tempting to claim that

$$
\begin{aligned}
\operatorname{sp}(H-E, P) & =\bigcup_{\mathbf{k} \in \mathbb{R}^{d}} \operatorname{sp}(H(\mathbf{k})-E) \times\{\mathbf{k}\}, \\
\operatorname{Exc}_{\mathrm{ess}} & =\left(\bigcup_{\mathbf{k} \in \mathbb{R}^{d}} \operatorname{sp}_{\mathrm{ess}}(H(\mathbf{k})-E) \times\{\mathbf{k}\}\right)^{\mathrm{cl}},
\end{aligned}
$$

where $\mathrm{sp}_{\mathrm{ess}}$ denotes the essential spectrum and the subscript cl denotes the closure. Unfortunately, at this level of generality there is a problem with (2.3) and (2.4). First of all, there is no guarantee that we can put the Lebesgue measure in (2.2). Secondly, the direct integral representation (2.2) is not defined uniquely, but only modulo sets of measure zero.

In concrete situations, however, (such as quasiparticle systems considered in Sect. 2.4) the direct integral (2.2) has an obvious distinguished realization involving the Lebesgue measure, for which the identities (2.3) and (2.4) are actually true. 


\subsection{Quasiparticle Quantum Systems}

Many important translation-invariant quantum systems can be described in terms of quasiparticles, that is, independent bosonic or fermionic modes with appropriately chosen dispersion relations (the dependence of the quasiparticle energy on the momentum).

Let us be more precise. For a Hilbert space $\mathcal{Z}$, the notation $\Gamma_{\mathrm{s}}(\mathcal{Z})$, resp. $\Gamma_{\mathrm{a}}(\mathcal{Z})$ will stand for the bosonic, resp. fermionic Fock space with the one particle space $\mathcal{Z}$.

By a quasiparticle quantum system we will mean $\left(H_{\mathrm{fr}}, P_{\mathrm{fr}}\right)$, where

$$
\begin{aligned}
H_{\mathrm{fr}} & =\sum_{i \in \mathcal{Q}} \int_{I_{i}} \omega_{i}(\mathbf{k}) b_{i}^{*}(\mathbf{k}) b_{i}(\mathbf{k}) \mathrm{d} \mathbf{k}, \\
P_{\mathrm{fr}} & =\sum_{i \in \mathcal{Q}} \int_{I_{i}} \mathbf{k} b_{i}^{*}(\mathbf{k}) b_{i}(\mathbf{k}) \mathrm{d} \mathbf{k},
\end{aligned}
$$

for some intervals $I_{i} \subset \mathbb{R}^{d}$, real continuous functions $I_{i} \ni \mathbf{k} \mapsto \omega_{i}(\mathbf{k})$, and creation, resp. annihilation operators $b_{i}^{*}(\mathbf{k})$ and $b_{i}(\mathbf{k})$. $\mathcal{Q}$ is called the set of quasiparticle species and it is partitioned into $\mathcal{Q}_{\mathrm{s}}$ and $\mathcal{Q}_{\mathrm{a}}$-bosonic and fermionic quasiparticles.

We are using the standard notation of the formalism of 2nd quantization: $b_{i}^{*}(\mathbf{k})$ and $b_{i}(\mathbf{k})$ satisfy the usual commutation/anticommutation relations. They are not true operators, only formal symbols, however the right hand sides of (2.5) and (2.6) are well defined as operators on the Fock space

$$
\underset{i \in \mathcal{Q}_{\mathrm{s}}}{\otimes} \Gamma_{\mathrm{s}}\left(L^{2}\left(I_{i}\right)\right) \otimes \underset{j \in \mathcal{Q}_{\mathrm{a}}}{\otimes} \Gamma_{\mathrm{a}}\left(L^{2}\left(I_{j}\right)\right)
$$

For $i \in \mathcal{Q}$, the set $I_{i}$ describes the allowed range of the momentum of a single $i$ th quasiparticle and $\omega_{i}(\mathbf{k})$ is its energy (dispersion relation) for momentum $\mathbf{k} \in \mathbb{R}^{d}$. Note that $I_{i}$ can be strictly smaller than $\mathbb{R}^{d}$-some quasiparticles may exist only for some momenta. This allows us more flexibility and is consistent with applications to condensed matter physics. It will be convenient to define

$$
I(\mathbf{k}):=\left\{i \in \mathcal{Q}: \mathbf{k} \in I_{i}(\mathbf{k})\right\}
$$

(the set of quasiparticles that may have momentum $\mathbf{k} \in \mathbb{R}^{d}$ ).

Clearly, if we know the dispersion relations $I_{i} \ni \mathbf{k} \mapsto \omega_{i}(\mathbf{k}), i \in \mathcal{Q}$, then we can determine the energy-momentum spectrum of $\left(H_{\mathrm{fr}}, P_{\mathrm{fr}}\right)$ :

$$
\begin{aligned}
& \operatorname{sp}\left(H_{\mathrm{fr}}, P_{\mathrm{fr}}\right)=\{(0, \mathbf{0})\} \\
& \quad \cup\left\{\left(\omega_{i_{1}}\left(\mathbf{k}_{1}\right)+\cdots+\omega_{i_{n}}\left(\mathbf{k}_{n}\right), \mathbf{k}_{1}+\cdots+\mathbf{k}_{n}\right): n=1,2,3 \ldots\right\}^{\mathrm{cl}} .
\end{aligned}
$$

Note that there is an obvious direct integral representation of the form (2.2) and the relations (2.3) and (2.4) hold. 


\subsection{Properties of the Excitation Spectrum of Quasiparticle Systems}

Let $(H, P)$ be a quasiparticle system. The energy-momentum spectrum of such systems has special properties. First, we have

$$
(0, \mathbf{0}) \in \operatorname{sp}(H, P),
$$

because of the Fock vacuum state, which is a unique joint eigenstate of $(H, P)$. Moreover, we have a remarkable addition property

$$
\mathrm{sp}(H, P)=\operatorname{sp}(H, P)+\mathrm{sp}(H, P) .
$$

Assume now that the Hamiltonian (2.5) is bounded from below, or what is equivalent, assume that all the dispersion relations are non-negative. Then the Fock vacuum is a ground state satisfying $E=0$, so that the excitation spectrum coincides with the energy-momentum spectrum. Thus we can rewrite (2.8) and (2.9) as

$$
\begin{aligned}
(0,0) & \in \operatorname{sp}(H-E, P), \\
\operatorname{sp}(H-E, P) & =\operatorname{sp}(H-E, P)+\operatorname{sp}(H-E, P) .
\end{aligned}
$$

Given (2.10), (2.11) is equivalent to

$$
\text { Exc } \supset \text { Exc + Exc. }
$$

Another remarkable property holds true if in addition the number of particle species is finite. We have then

$$
\operatorname{Exc}_{\mathrm{ess}}=(\mathrm{Exc}+\mathrm{Exc})^{\mathrm{cl}} \text {. }
$$

Indeed, using the continuity of the momentum spectrum, we easily see that only 1-particle states can belong to the discrete spectrum of the fiber Hamiltonians $H(\mathbf{k})$.

Before we proceed, let us introduce some terminology concerning real functions that will be useful in our study of quasiparticle-like spectra. Recall that a function $\mathbb{R}^{d} \ni \mathbf{k} \mapsto \epsilon(\mathbf{k})$ is called subadditive if

$$
\epsilon\left(\mathbf{k}_{1}+\mathbf{k}_{2}\right) \leq \epsilon\left(\mathbf{k}_{1}\right)+\epsilon\left(\mathbf{k}_{2}\right) .
$$

Let $\mathbb{R}^{d} \supset I \ni \mathbf{k} \mapsto \omega(\mathbf{k})$ be a given function. Define

$$
\begin{aligned}
\varsigma_{\omega}(\mathbf{k}) & =\inf \left\{\omega\left(\mathbf{k}_{1}\right)+\cdots+\omega\left(\mathbf{k}_{n}\right): \mathbf{k}_{1}+\cdots+\mathbf{k}_{n}=\mathbf{k}, \quad n=1,2,3, \ldots\right\}, \\
\varsigma_{\text {ess }, \omega}(\mathbf{k}) & =\inf \left\{\omega\left(\mathbf{k}_{1}\right)+\cdots+\omega\left(\mathbf{k}_{n}\right): \mathbf{k}_{1}+\cdots+\mathbf{k}_{n}=\mathbf{k}, \quad n=2,3, \ldots\right\},
\end{aligned}
$$

(By definition, the infimum of an empty set is $+\infty$ ). $\varsigma_{\omega}$ is known under the name of the subadditive hull of $\omega$. Equivalently, $\varsigma_{\omega}$ is the biggest subadditive function less than $\omega$.

Note the relation

$$
\varsigma_{\omega}(\mathbf{k})=\min \left\{\omega(\mathbf{k}), \varsigma_{\mathrm{ess}, \omega}(\mathbf{k})\right\} .
$$

Let us go back to a quasiparticle system (2.5), (2.6) with nonnegative dispersion relations. For $\mathbf{k} \in \mathbb{R}^{d}$, define

$$
\omega_{\min }(\mathbf{k}):=\min \left\{\omega_{i}: i \in I(\mathbf{k})\right\} .
$$


Recall the functions $\epsilon$ and $\epsilon_{\mathrm{ess}}$ and the parameters $\varepsilon$ and $c_{\mathrm{cr}}$ that we defined in Sects. 2.2 and 2.3 .

Theorem 2.1. 1. The bottom of the strict excitation spectrum is the subadditive hull of $\omega_{\min }$ :

$$
\epsilon(\mathbf{k})=\varsigma_{\omega_{\min }}(\mathbf{k}), \mathbf{k} \in \mathbb{R}^{d} .
$$

2. The energy gap satisfies

$$
\varepsilon=\inf _{\mathbf{k}} \omega_{\min }(\mathbf{k}) .
$$

3. The critical velocity satisfies

$$
c_{\mathrm{cr}}=\inf _{\mathbf{k} \neq \mathbf{0}} \frac{\omega_{\min }(\mathbf{k})}{|\mathbf{k}|}=\inf _{\mathbf{k} \neq \mathbf{0}} \frac{\epsilon_{\mathrm{ess}}(\mathbf{k})}{|\mathbf{k}|} .
$$

4. If in addition the number of quasiparticle species is finite, then

$$
\epsilon_{\mathrm{ess}}(\mathbf{k})=\varsigma_{\mathrm{ess}, \omega_{\min }}(\mathbf{k}), \quad \mathbf{k} \in \mathbb{R}^{d} .
$$

Note that we assume that the momentum space is $\mathbb{R}^{d}$. If we replace the momentum space $\mathbb{R}^{d}$ with $\frac{2 \pi}{L} \mathbb{Z}^{d}$ (that is, if we put our system on a torus of side length $L$ ) and we assume that all quasiparticles are bosonic, then all statements of this subsection generalize in an obvious way. However, because of the Pauli principle, not all of them generalize in the fermionic case.

\subsection{Approximate Versus Exact Quasiparticles}

One often considers quantum systems of the form

$$
H=H_{\text {fr }}+V, \quad P=P_{\text {fr }},
$$

where $\left(H_{\mathrm{fr}}, P_{\mathrm{fr}}\right)$ is a quasiparticle system and the perturbation $V$ is in some sense small. A description of physical systems in terms of approximate quasiparticles is very common in condensed matter physics. In particular, it appears naturally in the context of the so-called Hartree-Fock-Bogoliubov approximation, where one tries to optimize a quasiparticle description for a given quantum system [13].

Clearly, there is a considerable freedom in choosing the splitting of $H$ into $H_{\text {fr }}$ and $V$, and so quasiparticles of this kind are only vaguely determined. We will argue that in some cases a different concept of quasiparticles is useful, which is rigorous and in a way much more interesting. This concept is expressed in the following definition.

Let $(H, P)$ be a translation-invariant system on a Hilbert space $\mathcal{H}$. We will say that it is a quasiparticle-like system if it is unitarily equivalent to a quasiparticle system.

\subsection{Asymptotic Quasiparticles}

The above definition has one drawback. In practice we expect that the unitary equivalence mentioned in this definition is in some sense natural and constructed in the framework of scattering theory.

Scattering theory is quite far from the main subject of this paper, which is mostly concerned with purely spectral questions. However, since it has been 
mentioned and is very closely related to the concept of a quasiparticle, let us give a brief discussion of this topic.

For a number of many-body systems the basic idea of scattering theory can be described as follows. Using the evolution $\mathrm{e}^{\mathrm{i} t H}$ for $t \rightarrow \pm \infty$, we define two isometric operators

$$
S^{ \pm}: \underset{i \in \mathcal{Q}_{\mathrm{s}}}{\otimes} \Gamma_{\mathrm{s}}\left(L^{2}\left(I_{i}\right)\right) \otimes \underset{j \in \mathcal{Q}_{\mathrm{a}}}{\otimes} \Gamma_{\mathrm{a}}\left(L^{2}\left(I_{j}\right)\right) \rightarrow \mathcal{H}
$$

$S^{ \pm}$are called the wave or Møller operators and they satisfy

$$
H S^{ \pm}=S^{ \pm} H_{\mathrm{fr}}, \quad P S^{ \pm}=S^{ \pm} P_{\mathrm{fr}},
$$

where $\left(H_{\mathrm{fr}}, P_{\mathrm{fr}}\right)$ is a quasiparticle system. $S:=S^{+*} S^{-}$is then called the scattering operator.

We will say that the system is asymptotically complete if the wave operators $S^{ \pm}$are unitary. Clearly, if a system is asymptotically complete, then it is quasiparticle-like.

There are at least two classes of important physical system which possess a natural and rigorous scattering theory of this kind.

The first class consists of the 2nd quantization of Schrödinger many-body operators with two-body short range interactions [11]. One can show that these systems are asymptotically complete (see [10] and references therein). In this case the system is invariant wrt. the Galileian group and the dispersion relations have the form $\mathbb{R}^{d} \ni \mathbf{k} \mapsto E+\frac{\mathbf{k}^{2}}{2 m}$. Quasiparticles obtained in this context can be "elementary" - in applications to physics these are typically electrons and nuclei - as well as "composite" - atoms, ions, molecules, etc.

Another important class of systems where the concept of asymptotic quasiparticles has a rigorous foundation belongs to (relativistic) quantum field theory, as axiomatized by the Haag-Kastler or Wightman axioms. If we assume the existence of discrete mass shells, the so-called Haag-Ruelle theory allows us to construct the wave operators, see e.g., [17]. Note that in this case the system is covariant wrt. the Poincaré group and the dispersion relation has the form $\mathbb{R}^{d} \ni \mathbf{k} \mapsto \sqrt{m^{2}+\mathbf{k}^{2}}$. Here, quasiparticles are the usual stable particles.

Let us stress that both classes of systems can be interacting in spite of the fact that they are equivalent to free quasiparticle systems. In particular, their scattering operator can be nontrivial.

The above described classes of quantum systems are quite special. They are covariant wrt. rather large groups (Galilei or Poincaré) and have quite special dispersion relations.

\subsection{Quasiparticles in Condensed Matter Physics}

The concept of a quasiparticle is useful also in other contexts, without the Galilei or Poincaré covariance.

An interesting system which admits a quasiparticle interpretation is the free Fermi gas with a positive chemical potential. We describe this system in Sect. 2.16. In this case the scattering theory is trivial: $S^{+}=S^{-}$, and hence $S=11$. 
It seems that condensed matter physicists apply successfully the concept of a quasiparticle also to various interacting translation-invariant systems.

One class of such systems seems to be the Bose gas with repulsive interactions at zero temperature and positive density. In this case, apparently, the system is typically well described by a free Bose gas of quasiparticles of (at least) two kinds: at low momenta we have phonons with an approximately linear dispersion relation, and at somewhat higher momenta we have rotons. This idea underlies the famous Bogoliubov approximation [4], see also [8,14]. The phenomenon of superfluidity can be to a large extent explained within this picture. The model of free asymptotic phonons seems to work well in real experiments [18].

Another class of strongly interacting systems that seems to be successfully modeled by independent quasiparticles is the Fermi gas with attractive interactions at zero temperature and positive chemical potential. By using the Hartree-Fock-Bogoliubov (HFB) approach [19], which is closely related to the original Bardeen-Cooper-Schrieffer (BCS) approximation [1], one obtains a simple model that can be used to explain the superconductivity of the Fermi gas at very low temperatures. The corresponding quasiparticles are sometimes called partiholes.

Note that the above two examples - the interacting Bose and Fermi gasare neither Galilei nor Poincare covariant. This allows us to consider more general dispersion relations. However, we do not know whether these systems admit a quasiparticle interpretation or possess some kind of scattering theory. Unfortunately, rigorous results in this direction are rather modest. (There are attempts at scattering theory for some non-relativistic models of quantum field theory, see [12] and [15]. There exist also some results in a purely perturbative approach [20].)

\subsection{Quasiparticle-Like Excitation Spectrum}

The concept of a quasiparticle-like system, as defined in Sect. 2.6, is probably too strong for many applications. Let us propose a weaker property, which is more likely to be satisfied in various situations.

Again, our starting point is a translation-invariant system described by its Hamiltonian and momentum $(H, P)$. Let us assume that $H$ is bounded from below, with $E$, as usual, denoting the ground state energy. We will say that the excitation spectrum of $(H, P)$ is quasiparticle-like if it coincides with the excitation spectrum of a quasiparticle system [see (2.5) and (2.6)].

Clearly, the excitation spectrum of a quasiparticle-like system with a bounded from below Hamiltonian is quasiparticle-like. However, a system may have a quasiparticle-like excitation spectrum without being a quasiparticle-like system.

A quasiparticle-like excitation spectrum has special properties. In particular, it satisfies (2.10) and (2.11).

There exists a heuristic, but, we believe, a relatively convincing general argument why realistic translation-invariant quantum systems in thermodynamic limit at zero temperature should satisfy (2.10) and (2.11). It was 
essentially described at length in [8], but for the convenience of the reader we reproduce it here. Note in particular, that the infinite size of the quantum system plays an important role in this argument.

Consider a quantum gas in a box of a very large side length $L$, described by $\left(H^{L}, P^{L}\right)$. For shortness, let us drop the superscript $L$. First of all, it seems reasonable to assume that the system possesses a translation-invariant ground state, which we will denote by $\Phi$, so that $H \Phi=E \Phi, P \Phi=0$. Thus (2.10) holds.

Let $\left(E+e_{i}, \mathbf{k}_{i}\right) \in \operatorname{sp}(H, P), i=1,2$. We can find eigenvectors with these eigenvalues, that is, vectors $\Phi_{i}$ satisfying $H \Phi_{i}=\left(E+e_{i}\right) \Phi_{i}, P \Phi_{i}=\mathbf{k}_{i} \Phi_{i}$. Let us make the assumption that it is possible to find operators $A_{i}$ that are polynomials in creation and annihilation operator smeared with functions well localized in configuration space such that $P A_{i} \approx A_{i}\left(P+\mathbf{k}_{i}\right)$, and which approximately create the vectors $\Phi_{i}$ from the ground state, that is $\Phi_{i} \approx A_{i} \Phi$. (Note that here a large size of $L$ plays a role.) By replacing $\Phi_{2}$ with $\mathrm{e}^{\mathrm{iy} P} \Phi_{2}$ for some $\mathbf{y}$ and $A_{2}$ with $\mathrm{e}^{\mathrm{iy} P} A_{2} \mathrm{e}^{-\mathrm{iy} P}$, we can make sure that the regions of localization of $A_{1}$ and $A_{2}$ are separated by a large distance.

Now consider the vector $\Phi_{12}:=A_{1} A_{2} \Phi$. Clearly,

$$
P \Phi_{12} \approx\left(\mathbf{k}_{1}+\mathbf{k}_{2}\right) \Phi_{12} .
$$

$\Phi_{12}$ looks like the vector $\Phi_{i}$ in the region of localization of $A_{i}$, elsewhere it looks like $\Phi$. The Hamiltonian $H$ involves only expressions of short range (the potential decays in space). Therefore, we expect that

$$
H \Phi_{12} \approx\left(E+e_{1}+e_{2}\right) \Phi_{12} .
$$

If this is the case, it implies that $\left(E+e_{1}+e_{2}, \mathbf{k}_{1}+\mathbf{k}_{2}\right) \in \operatorname{sp}(H, P)$. Thus (2.12) holds.

\subsection{Bottom of a Quasiparticle-Like Excitation Spectrum}

Now suppose that $(H, P)$ is an arbitrary translation-invariant system with a bounded from below Hamiltonian. For simplicity, assume that its ground state energy is zero. We assume that we know its excitation $\operatorname{spectrum} \operatorname{sp}(H, P)$. There are two natural questions

1. Is $\operatorname{sp}(H, P)$ quasiparticle-like?

2. If it is the case, to what extent its dispersion relations are determined uniquely?

In order to give partial answers to the above questions, recall the functions $\epsilon$ and $\epsilon_{\mathrm{ess}}$, as well as the sets $\mathrm{Exc}_{\mathrm{d}}$ and Exc $\mathrm{ess}_{\text {en }}$ that we defined in Sects. 2.2 and 2.3 .

Theorem 2.2. Suppose that the excitation spectrum of $(H, P)$ is quasiparticlelike. Then the following is true:

1. $\epsilon$ is subadditive.

2. We can partly reconstruct some of the dispersion relations:

$$
\operatorname{Exc}_{\mathrm{d}}=\left\{\left(\omega_{i}(\mathbf{k}), \mathbf{k}\right): i \in \mathcal{Q}, \mathbf{k} \in \mathbb{R}^{d}\right\} \backslash \operatorname{Exc}_{\mathrm{ess}} .
$$


Consequently, for $\mathbf{k}$ satisfying $\epsilon(\mathbf{k})<\epsilon_{\mathrm{ess}}(\mathbf{k})$,

$$
\epsilon(\mathbf{k})=\omega_{\min }(\mathbf{k}),
$$

where $\omega_{\min }$ was defined in (2.14).

3. If the number of quasiparticles species is finite, we can reconstruct $\epsilon_{\mathrm{ess}}$ from $\epsilon$ :

$$
\epsilon_{\text {ess }}(\mathbf{k})=\inf \left\{\epsilon\left(\mathbf{k}_{1}\right)+\epsilon\left(\mathbf{k}_{2}\right): \mathbf{k}=\mathbf{k}_{1}+\mathbf{k}_{2}\right\} .
$$

The existential part of the inverse problem has a partial solution:

Theorem 2.3. Suppose that $\mathbb{R}^{d} \ni \mathbf{k} \mapsto \omega(\mathbf{k})$ be a given subadditive function. Consider the translation-invariant system

$$
H_{\text {fr }}=\int \omega(\mathbf{k}) b_{\mathbf{k}}^{*} b_{\mathbf{k}} \mathrm{d} \mathbf{k}, \quad P_{\mathrm{fr}}=\int \mathbf{k} b_{\mathbf{k}}^{*} b_{\mathbf{k}} \mathrm{d} \mathbf{k} .
$$

Then

$$
\begin{aligned}
\epsilon(\mathbf{k}) & =\omega(\mathbf{k}), \\
\epsilon_{\mathrm{ess}}(\mathbf{k}) & =\inf \left\{\omega\left(\mathbf{k}_{1}\right)+\omega\left(\mathbf{k}_{2}\right): \mathbf{k}=\mathbf{k}_{1}+\mathbf{k}_{2}\right\} .
\end{aligned}
$$

The answer to the uniqueness part of the inverse problem is negative. The only situation where we can identify dispersion relations from the spectral information involves $\mathrm{Exc}_{\mathrm{d}}$, see (2.17). The following example shows that we have quite a lot of freedom in choosing a dispersion relation giving a prescribed excitation spectrum. For instance, all the Hamiltonians below have the same excitation spectrum and essential excitation spectrum with $\epsilon(\mathbf{k})=$ $\epsilon_{\mathrm{ess}}(\mathbf{k})=|\mathbf{k}|$ :

$$
H=\int_{|\mathbf{k}|<c}|\mathbf{k}|\left(1+d|\mathbf{k}|^{\alpha}\right) b_{\mathbf{k}}^{*} b_{\mathbf{k}} \mathrm{d} \mathbf{k},
$$

where $c>0, d \geq 0$ and $\alpha>0$ are arbitrary.

\subsection{Translation-Invariant Systems with Two Superselection Sectors}

Suppose that a Hilbert space $\mathcal{H}$ has a decomposition $\mathcal{H}=\mathcal{H}^{+} \oplus \mathcal{H}^{-}$, which can be treated as a superselection rule. This means that all observables decompose into direct sums. In particular, the Hamiltonian and momentum decompose as $(H, P)=\left(H^{+}, P^{+}\right) \oplus\left(H^{-}, P^{-}\right)$. Clearly,

$$
\operatorname{sp}(H, P)=\operatorname{sp}\left(H^{+}, P^{+}\right) \cup \operatorname{sp}\left(H^{-}, P^{-}\right) .
$$

We will often assume that $H$ is bounded from below and possesses a translation-invariant ground state $\Phi$ with energy $E$, which belongs to the sector $\mathcal{H}^{+}$. The sector $\mathcal{H}^{+}$will be called even. The other sector $\mathcal{H}^{-}$will be called odd.

Under these assumptions we will call $\operatorname{sp}\left(H^{+}-E, P^{+}\right)$, resp. $\operatorname{sp}\left(H^{-}-\right.$ $\left.E, P^{-}\right)$the even, resp. odd excitation spectrum. We introduce also the strict even excitation spectrum:

$$
\operatorname{Exc}^{+}:=\operatorname{sp}\left(\left.\left(H^{+}-E, P^{+}\right)\right|_{\{\Phi\}^{\perp}}\right)
$$

The strict odd excitation spectrum will coincide with the full odd excitation spectrum: 


$$
\operatorname{Exc}^{-}:=\operatorname{sp}\left(H^{-}-E, P^{-}\right) .
$$

Finally, we define the even and odd essential excitation spectrum Exc ess $^{ \pm}$ just as in Sect. 2.3, except that we replace $(H, P)$ with $\left(H^{ \pm}, P^{ \pm}\right)$. Exc $_{\mathrm{ess}}^{ \pm}$:

We introduce also a special notation for the bottom of the sets $\mathrm{Exc}^{ \pm}$and

$$
\begin{aligned}
\epsilon^{ \pm}(\mathbf{k}) & :=\inf \left\{e:(e, \mathbf{k}) \in \operatorname{Exc}^{ \pm}\right\} \\
\epsilon_{\mathrm{ess}}^{ \pm}(\mathbf{k}) & :=\inf \left\{e:(e, \mathbf{k}) \in \operatorname{Exc}_{\mathrm{ess}}^{ \pm}\right\} .
\end{aligned}
$$

Clearly,

$$
\begin{aligned}
\operatorname{sp}(H-E, P) & =\operatorname{sp}\left(H^{+}-E, P^{+}\right) \cup \operatorname{sp}\left(H^{-}-E, P^{-}\right), \\
\operatorname{Exc} & =\operatorname{Exc}^{+} \cup \operatorname{Exc}^{-}, \\
\operatorname{Exc}_{\mathrm{ess}} & =\operatorname{Exc}_{\mathrm{ess}}^{+} \cup \operatorname{Exc}_{\mathrm{ess}}^{-}, \\
\epsilon(\mathbf{k}) & =\min \left\{\epsilon^{-}(\mathbf{k}), \epsilon^{+}(\mathbf{k})\right\}, \\
\epsilon_{\mathrm{ess}}(\mathbf{k}) & =\min \left\{\epsilon_{\mathrm{ess}}^{-}(\mathbf{k}), \epsilon_{\mathrm{ess}}^{+}(\mathbf{k})\right\} .
\end{aligned}
$$

\subsection{Quasiparticle Systems with the Fermionic Superselection Rule}

Consider a quasiparticle system $\left(H_{\mathrm{fr}}, P_{\mathrm{fr}}\right)$ on the Fock space $(2.7)$. Define the fermionic number operator as

$$
N_{\mathrm{a}}=\sum_{i \in \mathcal{Q}_{\mathrm{a}}} b_{i}^{*}(\mathbf{k}) b_{i}(\mathbf{k}) .
$$

Clearly, the fermionic parity $(-1)^{N_{a}}$ provides a natural superselection rule. If $\mathcal{H}=\mathcal{H}^{+} \oplus \mathcal{H}^{-}$denotes the corresponding direct sum decomposition, then the Hamiltonian and momentum decompose as

$$
\left(H_{\mathrm{fr}}, P_{\mathrm{fr}}\right)=\left(H_{\mathrm{fr}}^{+}, P_{\mathrm{fr}}^{+}\right) \oplus\left(H_{\mathrm{fr}}^{-}, P_{\mathrm{fr}}^{-}\right) .
$$

(2.27) will be called a two-sector quasiparticle system.

Clearly, if we know the dispersion relations $I_{i} \ni \mathbf{k} \mapsto \omega_{i}(\mathbf{k}), i \in \mathcal{Q}$, then we can determine the even and odd energy momentum spectrum of $\left(H_{\mathrm{fr}}^{+}, P_{\mathrm{fr}}^{+}\right)$:

$$
\begin{aligned}
\operatorname{sp}\left(H_{\mathrm{fr}}^{+}, P_{\mathrm{fr}}^{+}\right)=\{ & (0,0)\} \\
\cup\left\{\left(\omega_{i_{1}}\left(\mathbf{k}_{1}\right)+\cdots+\omega_{i_{n}}\left(\mathbf{k}_{n}\right), \mathbf{k}_{1}+\cdots+\mathbf{k}_{n}\right):\right. & \\
& \quad \text { even number of fermions, } n=1,2,3 \ldots\}^{\mathrm{cl}}, \\
\operatorname{sp}\left(H_{\mathrm{fr}}^{-}, P_{\mathrm{fr}}^{-}\right)=\left\{\left(\omega_{i_{1}}\left(\mathbf{k}_{1}\right)+\cdots+\omega_{i_{n}}\left(\mathbf{k}_{n}\right), \mathbf{k}_{1}+\cdots+\mathbf{k}_{n}\right):\right. & \quad \text { odd number of fermions, } n=1,2,3 \ldots\}^{\mathrm{cl}} .
\end{aligned}
$$

\subsection{Properties of the Excitation Spectrum of Two-Sector Quasiparticle Systems}

Let $(H, P)=\left(H^{+}, P^{+}\right) \oplus\left(H^{-}, P^{-}\right)$be a two-sector quasiparticle system. Clearly, we have

$$
(0, \mathbf{0}) \in \operatorname{sp}\left(H^{+}, P^{+}\right)
$$


because of the Fock vacuum. Here are the properties of the even and odd excitation spectrum:

$$
\begin{aligned}
\operatorname{sp}\left(H^{+}, P^{+}\right) & =\operatorname{sp}\left(H^{+}, P^{+}\right)+\operatorname{sp}\left(H^{+}, P^{+}\right) \\
& \supset \operatorname{sp}\left(H^{-}, P^{-}\right)+\operatorname{sp}\left(H^{-}, P^{-}\right), \\
\operatorname{sp}\left(H^{-}, P^{-}\right) & =\operatorname{sp}\left(H^{-}, P^{-}\right)+\operatorname{sp}\left(H^{+}, P^{+}\right) .
\end{aligned}
$$

Assume now that the Hamiltonian is bounded from below. Then the Fock vacuum is a translation-invariant ground state satisfying $E=0$, so that the excitation spectrum coincides with the energy-momentum spectrum. Thus we can rewrite (2.28)-(2.31) as

$$
\begin{aligned}
(0,0) & \in \operatorname{sp}\left(H^{+}-E, P^{+}\right), \\
\operatorname{sp}\left(H^{+}-E, P^{+}\right) & =\operatorname{sp}\left(H^{+}-E, P^{+}\right)+\operatorname{sp}\left(H^{+}-E, P^{+}\right) \\
& \supset \operatorname{sp}\left(H^{-}-E, P^{-}\right)+\operatorname{sp}\left(H^{-}-E, P^{-}\right), \\
\operatorname{sp}\left(H^{-}-E, P^{-}\right) & =\operatorname{sp}\left(H^{-}-E, P^{-}\right)+\operatorname{sp}\left(H^{+}-E, P^{+}\right) .
\end{aligned}
$$

Given (2.32), (2.33)-(2.35) are equivalent to

$$
\begin{aligned}
& \operatorname{Exc}^{+} \supset\left(\mathrm{Exc}^{+}+\mathrm{Exc}^{+}\right) \cup\left(\mathrm{Exc}^{-}+\mathrm{Exc}^{-}\right), \\
& \mathrm{Exc}^{-} \supset \mathrm{Exc}^{-}+\mathrm{Exc}^{+} .
\end{aligned}
$$

If in addition the number of particle species is finite, then

$$
\begin{aligned}
& \operatorname{Exc}_{\mathrm{ess}}^{+}=\left(\mathrm{Exc}^{+}+\mathrm{Exc}^{+}\right)^{\mathrm{cl}} \cup\left(\mathrm{Exc}^{-}+\mathrm{Exc}^{-}\right)^{\mathrm{cl}}, \\
& \operatorname{Exc}_{\mathrm{ess}}^{-}=\left(\mathrm{Exc}^{-}+\mathrm{Exc}^{+}\right)^{\mathrm{cl}} .
\end{aligned}
$$

\subsection{Two-Sector Quasiparticle-Like Spectrum}

Consider now an arbitrary translation-invariant system with two superselection sectors $(H, P)=\left(H^{+}, P^{+}\right) \oplus\left(H^{-}, P^{-}\right)$. We will assume that $H$ is bounded from below and the ground state with energy $E$ is translation invariant and belongs to the sector $\mathcal{H}^{+}$.

We will say that the excitation spectrum of $\left(H^{+}, P^{+}\right) \oplus\left(H^{-}, P^{-}\right)$is two-sector quasiparticle-like if it coincides with the excitation spectrum of a two-sector quasiparticle system. Such an excitation spectrum has special properties. In particular, it satisfies (2.32)-(2.35).

There exists a heuristic general argument why realistic translation-invariant quantum systems in thermodynamic limit should satisfy (2.32)-(2.35). It is an obvious modification of the argument given in Sect. 2.9.

Indeed, we need to notice what follows. $(-1)^{N_{\mathrm{a}}}$ is always a superselection rule for realistic quantum system. In particular, if we assume that the ground state is nondegenerate, it has to be either bosonic or fermionic. We make an assumption that it is bosonic.

The eigenvectors $\Phi_{1}$ and $\Phi_{2}$, discussed in Sect. 2.9, can be chosen to be purely bosonic or fermionic. Using the fact that the ground state is purely bosonic, we see that we can chose the operators $A_{1}$ and $A_{2}$ to be purely bosonic 
or fermionic. (That means, they either commute or anticommute with $(-1)^{N_{\mathrm{a}}}$ ). Consequently, we have the following possibilities:

- Both $\Phi_{1}$ and $\Phi_{2}$ are bosonic. Then $\Phi_{12}$ is bosonic.

- Both $\Phi_{1}$ and $\Phi_{2}$ are fermionic. Then $\Phi_{12}$ is bosonic.

- One of $\Phi_{1}$ and $\Phi_{2}$ is bosonic, the other is fermionic. Then $\Phi_{12}$ is fermionic.

This implies (2.36) and (2.37).

\subsection{Bottom of a Two-Sector Quasiparticle-Like Excitation Spectrum}

Suppose again that $(H, P)=\left(H^{+}, P^{+}\right) \oplus\left(H^{-}, P^{-}\right)$is a translation-invariant system with two superselection sectors. We assume that we know its excitation spectrum. We would like to describe some criteria to verify whether it is two-sector quasiparticle-like. These criteria will involve the properties of the bottom of the even and odd excitation spectrum.

Theorem 2.4. Suppose that the excitation spectrum of $\left(H^{+}, P^{+}\right) \oplus\left(H^{-}, P^{-}\right)$ is two-sector quasiparticle-like.

1. We have the following subadditivity properties:

$$
\begin{aligned}
& \epsilon^{-}\left(\mathbf{k}_{1}+\mathbf{k}_{2}\right) \leq \epsilon^{-}\left(\mathbf{k}_{1}\right)+\epsilon^{+}\left(\mathbf{k}_{2}\right), \\
& \epsilon^{+}\left(\mathbf{k}_{1}+\mathbf{k}_{2}\right) \leq \epsilon^{-}\left(\mathbf{k}_{1}\right)+\epsilon^{-}\left(\mathbf{k}_{2}\right), \\
& \epsilon^{+}\left(\mathbf{k}_{1}+\mathbf{k}_{2}\right) \leq \epsilon^{+}\left(\mathbf{k}_{1}\right)+\epsilon^{+}\left(\mathbf{k}_{2}\right) .
\end{aligned}
$$

2. If the number of species of quasiparticles is finite, then we can reconstruct $\epsilon_{\mathrm{ess}}^{-}$and $\epsilon_{\mathrm{ess}}^{+}$from $\epsilon^{-}$and $\epsilon^{+}$:

$$
\begin{array}{ll}
\epsilon_{\mathrm{ess}}^{-}(\mathbf{k})=\inf \left\{\epsilon^{-}\left(\mathbf{k}_{1}\right)+\epsilon^{+}\left(\mathbf{k}_{2}\right): \mathbf{k}=\mathbf{k}_{1}+\mathbf{k}_{2}\right\} \\
\epsilon_{\mathrm{ess}}^{+}(\mathbf{k})=\inf \left\{\epsilon^{+}\left(\mathbf{k}_{1}\right)+\epsilon^{+}\left(\mathbf{k}_{2}\right),\right. & \left.\epsilon^{-}\left(\mathbf{k}_{1}\right)+\epsilon^{-}\left(\mathbf{k}_{2}\right): \mathbf{k}=\mathbf{k}_{1}+\mathbf{k}_{2}\right\} .
\end{array}
$$

\subsection{Non-Interacting Fermi Gas}

Let us give a brief discussion of the free Fermi gas with chemical potential $\mu$ in $d$ dimensions. For simplicity, we will assume that particles have no internal degrees of freedom such as spin.

The Hilbert space of $n$ fermions equals $\Gamma_{\mathrm{a}}^{n}\left(L^{2}\left(\mathbb{R}^{d}\right)\right.$ ) (antisymmetric square integrable functions on $\left.\left(\mathbb{R}^{d}\right)^{n}\right)$. Let $\Delta_{(i)}$ denote the Laplacian $\Delta$ acting on the $i$ th variable. Then the Hamiltonian equals

$$
H^{n}=\sum_{i=1}^{n}\left(-\Delta_{(i)}-\mu\right) .
$$

It commutes with the momentum operator

$$
P^{n}:=\sum_{i=1}^{n}-\mathrm{i} \nabla_{(i)} .
$$

It is convenient to put together various $n$-particle sectors in a single Fock space

$$
\Gamma_{\mathrm{a}}\left(L^{2}(\Lambda)\right):=\bigoplus_{n=0}^{\infty} \Gamma_{\mathrm{a}}^{n}\left(L^{2}(\Lambda)\right)
$$


Then the basic observables are the Hamiltonian, the total momentum and the number operator:

$$
\begin{aligned}
& H=\bigoplus_{n=0}^{\infty} H^{n}=\int a_{\mathbf{x}}^{*}(-\Delta-\mu) a_{\mathbf{x}} \mathrm{d} \mathbf{x}, \\
& P=\bigoplus_{n=0}^{\infty} P^{n}=-\mathrm{i} \int a_{\mathbf{x}}^{*} \nabla_{\mathbf{x}} a_{\mathbf{x}} \mathrm{d} \mathbf{x}, \\
& N=\bigoplus_{n=0}^{\infty} n=\int a_{\mathbf{x}}^{*} a_{\mathbf{x}} \mathrm{d} \mathbf{x},
\end{aligned}
$$

where $a_{\mathbf{x}}^{*} / a_{\mathbf{x}}$ are the usual fermionic creation/annihilation operators.

The three operators in (2.41) describe only a finite number of particles in an infinite space. We would like to investigate homogeneous Fermi gas at a positive density in the thermodynamic limit. Following the accepted, although somewhat unphysical tradition, we first consider our system on $\Lambda=$ $[-L / 2, L / 2]^{d}$, the $d$-dimensional cubic box of side length $L$, with periodic boundary conditions. Note that the spectrum of the momentum becomes $\frac{2 \pi}{L} \mathbb{Z}^{d}$. At the end we let $L \rightarrow \infty$.

It is convenient to pass to the momentum representation:

$$
\begin{aligned}
H^{L} & =\sum_{\mathbf{k}}\left(\mathbf{k}^{2}-\mu\right) a_{\mathbf{k}}^{*} a_{\mathbf{k}} \\
P^{L} & =\sum_{\mathbf{k}} \mathbf{k} a_{\mathbf{k}}^{*} a_{\mathbf{k}}, \\
N^{L} & =\sum_{\mathbf{k}} a_{\mathbf{k}}^{*} a_{\mathbf{k}},
\end{aligned}
$$

where we used (2.41) and $a_{\mathbf{x}}=L^{-d / 2} \sum_{\mathbf{k}} \mathrm{e}^{\mathrm{i} \mathbf{k x}} a_{\mathbf{k}}$. We sum over $\mathbf{k} \in \frac{2 \pi}{L} \mathbb{Z}^{d}$.

It is natural to change the representation of canonical anticommutation relations and replace the usual fermionic creation/annihilation operators by new ones, which kill the ground state of the Hamiltonian:

$$
\begin{array}{ll}
b_{\mathbf{k}}^{*}:=a_{\mathbf{k}}^{*}, & b_{\mathbf{k}}:=a_{\mathbf{k}}, \quad \mathbf{k}^{2}>\mu, \\
b_{\mathbf{k}}^{*}:=a_{\mathbf{k}}, & b_{\mathbf{k}}:=a_{\mathbf{k}}^{*}, \quad \mathbf{k}^{2} \leq \mu .
\end{array}
$$

Then,

$$
\begin{aligned}
H^{L} & =\sum_{\mathbf{k}}\left|\mathbf{k}^{2}-\mu\right| b_{\mathbf{k}}^{*} b_{\mathbf{k}}+E^{L}, \\
P^{L} & =\sum_{\mathbf{k}} \mathbf{k} b_{\mathbf{k}}^{*} b_{\mathbf{k}}, \\
N^{L} & =\sum_{\mathbf{k}} \operatorname{sgn}\left(\mathbf{k}^{2}-\mu\right) b_{\mathbf{k}}^{*} b_{\mathbf{k}}+C^{L},
\end{aligned}
$$

where

$$
\begin{aligned}
E^{L} & =\sum_{\mathbf{k}^{2} \leq \mu}\left(\mathbf{k}^{2}-\mu\right), \\
C^{L} & =\sum_{\mathbf{k}^{2} \leq \mu} 1 .
\end{aligned}
$$


It is customary to drop the constants $E^{L}$ and $C^{L}$.

Set (temporarily) $\omega(\mathbf{k})=\left|\mathbf{k}^{2}-\mu\right|$. In the case of an infinite space, the above analysis suggests that it is natural to postulate

$$
\begin{aligned}
H & =\int \omega(\mathbf{k}) b_{\mathbf{k}}^{*} b_{\mathbf{k}} \mathrm{d} \mathbf{k}, \\
P & =\int \mathbf{k} b_{\mathbf{k}}^{*} b_{\mathbf{k}} \mathrm{d} \mathbf{k}, \\
N & =\int \operatorname{sgn}\left(\mathbf{k}^{2}-\mu\right) b_{\mathbf{k}}^{*} b_{\mathbf{k}} \mathrm{d} \mathbf{k},
\end{aligned}
$$

as the Hamiltonian, total momentum and number operator of the free Fermi gas from the beginning, instead of (2.41).

The operators $b_{\mathbf{k}}^{*} / b_{\mathbf{k}}$ can be called quasiparticle creation/annihilation operators and the function $\mathbf{k} \mapsto \omega(\mathbf{k})$ the quasiparticle dispersion relation. Thus a quasiparticle is a true particle above the Fermi level and a hole below the Fermi level.

In Sect. 4 we describe a version of the BCS theory based on the HartreeFock-Bogoliubov approximation. This approximation suggests that the interacting Fermi gas can be described, at least approximately, by a Hamiltonian of the form (2.43) with a dispersion relation $\mathbf{k} \mapsto \omega(\mathbf{k})$ that resembles $\left|\mathbf{k}^{2}-\mu\right|$, except that its minimum is strictly positive.

\subsection{Examples of the Energy-Momentum Spectrum}

The energy-momentum spectrum of a Fermi gas described by (2.43) and (2.44) with various dispersion relations $\omega$ can sometimes have a curious shape. In the remaining part of this section we will illustrate this with several examples. We will present diagrams representing the energy-momentum spectrum. In the full and the odd cases, the dispersion relation $\omega$ is a singular part of the spectrum and it will be denoted by a solid line. In the even case, the dispersion relation will be denoted by a dotted line. We will always consider the spherically symmetric case.

First consider the non-interacting Fermi gas, which, as we argued above, has the dispersion relation $\omega(\mathbf{k})=\left|\mathbf{k}^{2}-\mu\right|$. In dimension 1 its energy-momentum spectrum looks quite interesting (Figs. 1, 2, 3).

Clearly, for $d \geq 2$ the energy-momentum spectrum is rather boring (Fig. 4).

In the case of an interacting Fermi gas, we assume that

$$
\omega=\sqrt{\left(\mathbf{k}^{2}-\mu\right)^{2}+\gamma^{2}} .
$$

Calculations presented in Sect. 4, in particular (4.16), suggest that the dispersion relation obtained by the HFB method is qualitatively similar to (2.46) (Figs. 5, 6, 7, 8, 9, 10).

Again, the case $d=1$ differs from $d \geq 2$. However, in all dimensions the energy gap and the critical velocity are strictly positive. 


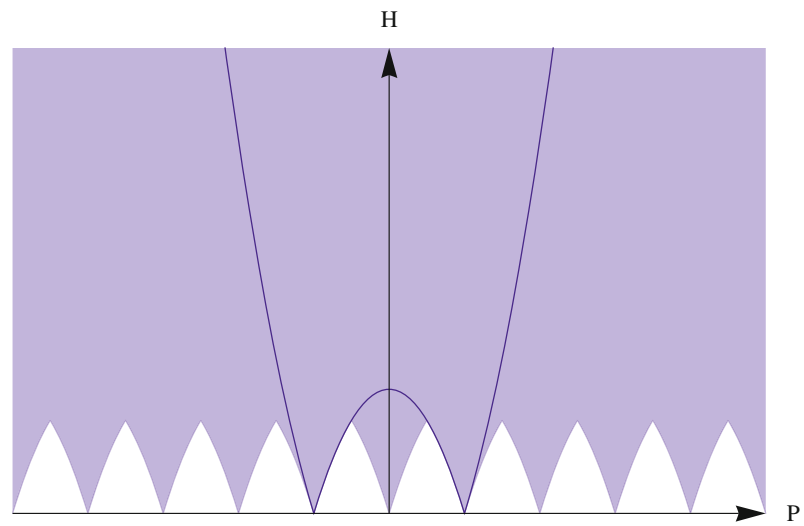

Figure $1 . \operatorname{sp}(H, P)$ in the non-interacting case, $d=1$

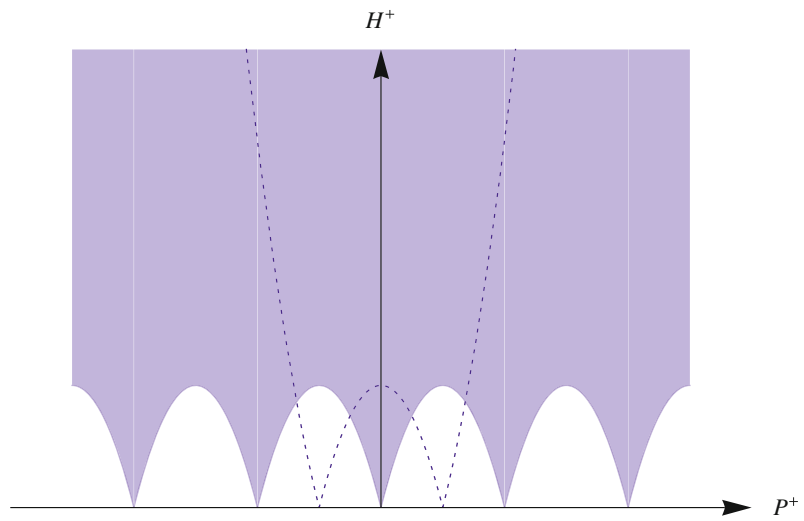

Figure 2. $\operatorname{sp}\left(H^{+}, P^{+}\right)$in the non-interacting case, $d=1$

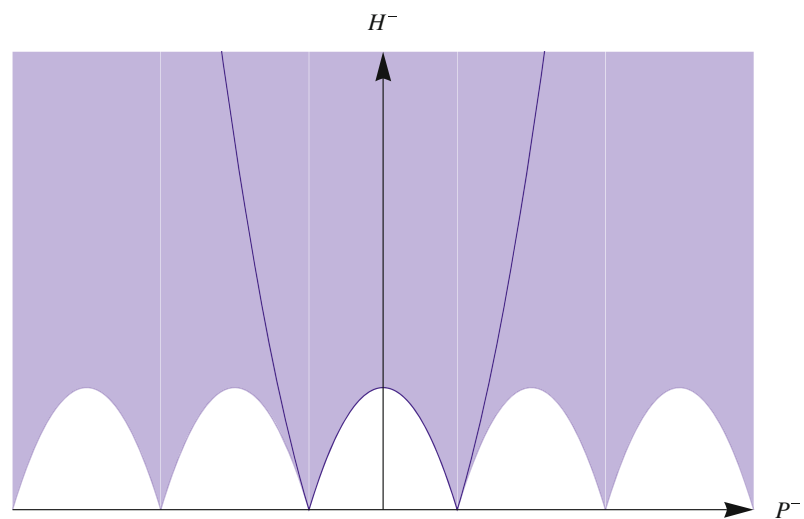

Figure 3. $\operatorname{sp}\left(H^{-}, P^{-}\right)$in the non-interacting case, $d=1$ 
$\mathrm{H}$

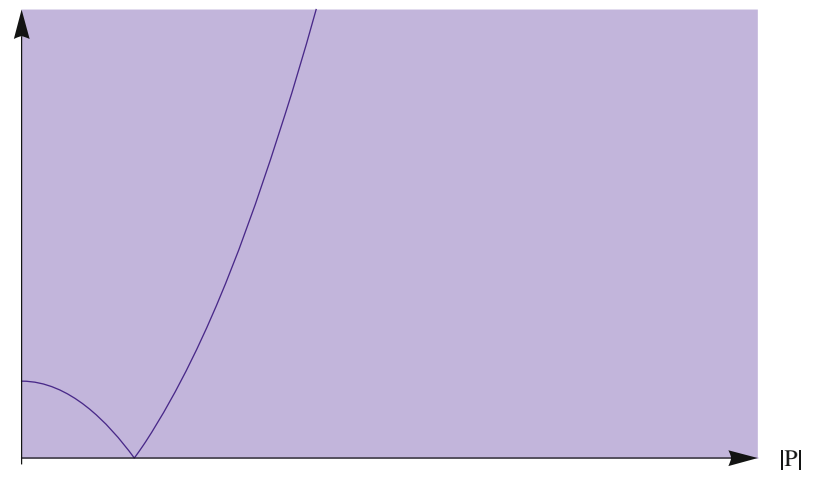

Figure 4. $\operatorname{sp}(H, P), \operatorname{sp}\left(H^{+}, P^{+}\right), \operatorname{sp}\left(H^{-}, P^{-}\right)$in the noninteracting case, $d \geq 2$

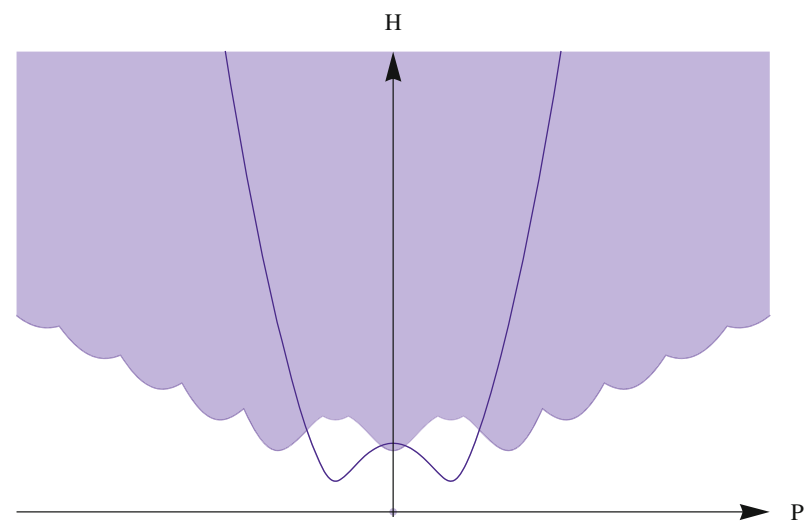

Figure 5. $\operatorname{sp}(H, P)$ in the interacting case, $d=1$

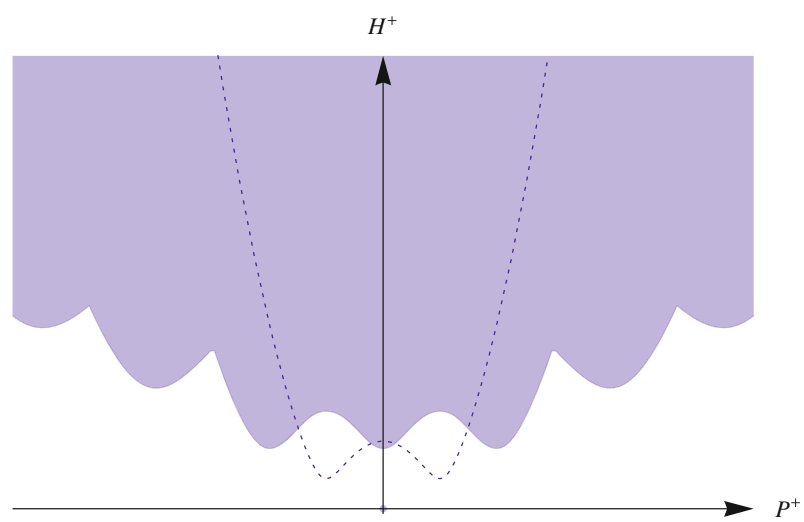

Figure $6 . \operatorname{sp}\left(H^{+}, P^{+}\right)$in the interacting case, $d=1$ 


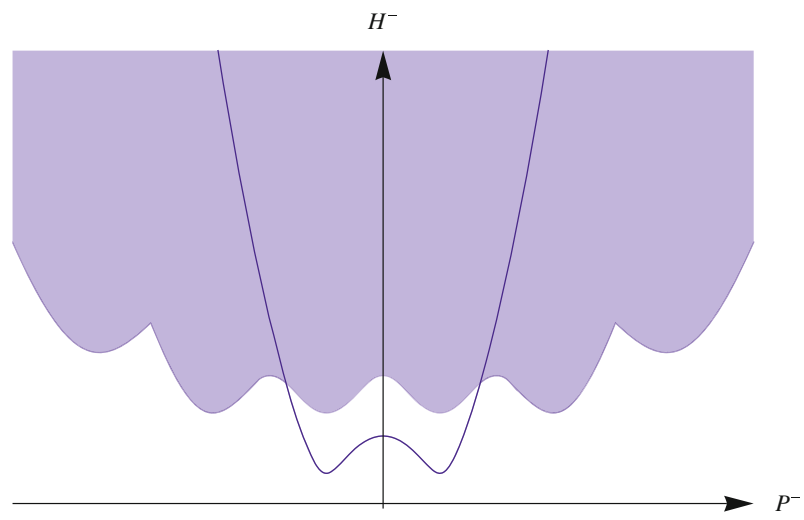

Figure $7 . \operatorname{sp}\left(H^{-}, P^{-}\right)$in the interacting case, $d=1$ $\mathrm{H}$

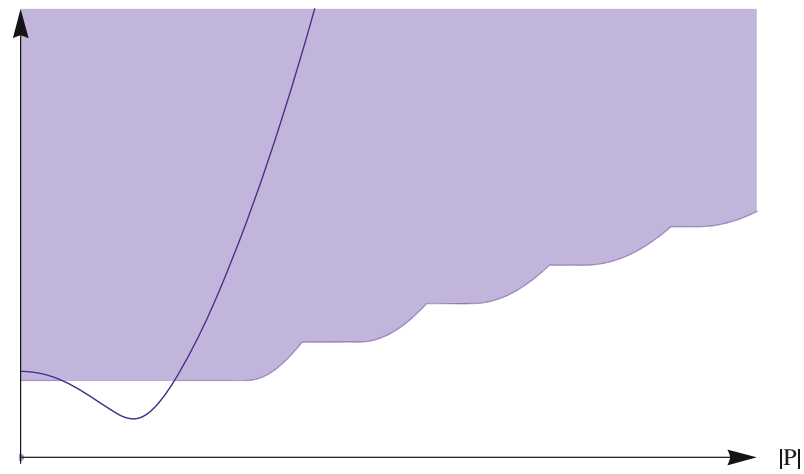

Figure $8 . \operatorname{sp}(H, P)$ in the interacting case, $d \geq 2$

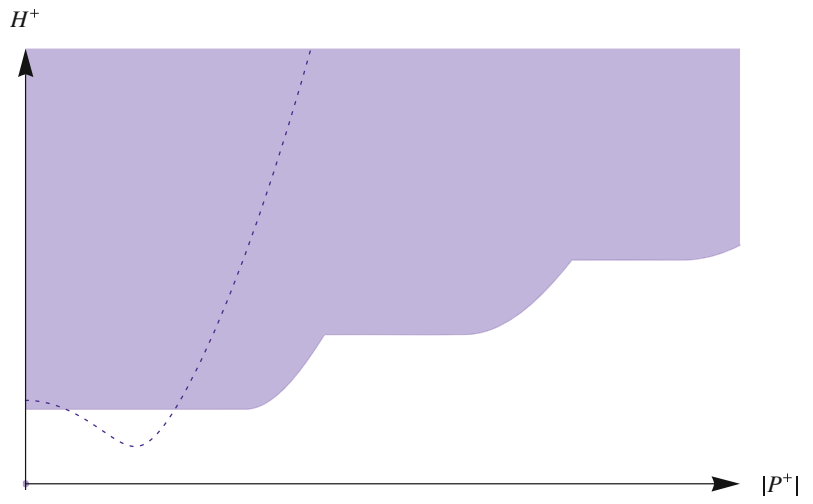

Figure $9 . \operatorname{sp}\left(H^{+}, P^{+}\right)$in the interacting case, $d \geq 2$ 


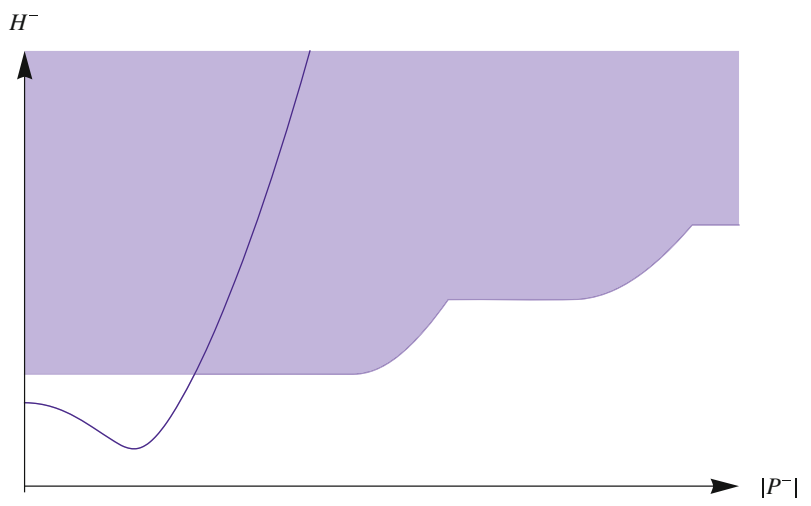

Figure 10. $\operatorname{sp}\left(H^{-}, P^{-}\right)$in the interacting case, $d \geq 2$

\section{The Model and Conjectures}

In this section we describe some classes of interacting models that seem to be relevant for condensed matter physics. We also formulate conjectures about these models that we think are suggested by "physical intuition".

Internal degrees of freedom of particles, such as spin, play an important role in fermionic systems. They are in particular crucial in the BCS approach. Therefore, we will take them into account. We will assume that they are described by a finite dimensional Hilbert space $\mathbb{C}^{m}$. Thus the one-particle space of the system is $L^{2}\left(\mathbb{R}^{d}, \mathbb{C}^{m}\right)$.

\subsection{1-Particle Energy}

The kinetic energy of one particle including its chemical potential is given by a self-adjoint operator $T$ on $L^{2}\left(\mathbb{R}^{d}, \mathbb{C}^{m}\right)$. We use the following notation for its integral kernel: for $\Phi \in L^{2}\left(\mathbb{R}^{d}, \mathbb{C}^{m}\right)$,

$$
(T \Phi)_{i_{1}}\left(\mathbf{x}_{1}\right)=\sum_{i_{2}} \int T_{i_{1}, i_{2}}\left(\mathbf{x}_{1}, \mathbf{x}_{2}\right) \Phi_{i_{2}}\left(\mathbf{x}_{2}\right) \mathrm{d} \mathbf{x}_{2} .
$$

We assume that $T$ is a self-adjoint and translation-invariant one-body operator. Clearly,

$$
\begin{aligned}
T_{i_{1}, i_{2}}\left(\mathbf{x}_{1}, \mathbf{x}_{2}\right) & =\overline{T_{i_{2}, i_{1}}\left(\mathbf{x}_{2}, \mathbf{x}_{1}\right)} \\
& =T_{i_{1}, i_{2}}\left(\mathbf{x}_{1}+\mathbf{y}, \mathbf{x}_{2}+\mathbf{y}\right) .
\end{aligned}
$$

The first identity expresses the hermiticity and the second the translation invariance of $T$.

We will sometimes assume that $T$ is real, that is, invariant with respect to the complex conjugation. This means that $T_{i_{1}, i_{2}}\left(\mathbf{x}_{1}, \mathbf{x}_{2}\right)$ are real. An example of a real 1-particle energy is

$$
T_{i j}=\left(-\frac{1}{2 m_{i}} \Delta-\mu_{i}\right) \delta_{i, j},
$$

where the $i$ th "spin" has the mass $m_{i}$ and the chemical potential $\mu_{i}$. 
If the operator $T$ has the form

$$
T_{i, j}\left(\mathbf{x}_{i}, \mathbf{x}_{j}\right)=t\left(\mathbf{x}_{i}, \mathbf{x}_{j}\right) \delta_{i, j}
$$

for some function $t$ satisfying

$$
\begin{aligned}
t\left(\mathbf{x}_{1}, \mathbf{x}_{2}\right) & =\overline{t\left(\mathbf{x}_{2}, \mathbf{x}_{1}\right)} \\
& =t\left(\mathbf{x}_{1}+\mathbf{y}, \mathbf{x}_{2}+\mathbf{y}\right)
\end{aligned}
$$

then we will say that $T$ is spin-independent.

Clearly, the 1-particle energy can be written as

$$
T_{i, j}\left(\mathbf{x}_{1}, \mathbf{x}_{2}\right)=(2 \pi)^{-d} \int \tau_{i, j}(\mathbf{k}) \mathrm{e}^{\mathrm{i} \mathbf{k}\left(\mathbf{x}_{1}-\mathbf{x}_{2}\right)} \mathrm{d} \mathbf{k} .
$$

If it is real, then $\tau_{i, j}(\mathbf{k})=\tau_{i, j}(-\mathbf{k})$

If it is spin independent, then

$$
\tau_{i, j}(\mathbf{k})=\tau(\mathbf{k}) \delta_{i, j} .
$$

In the real spin-independent case we have $\tau(\mathbf{k})=\tau(-\mathbf{k})$.

\subsection{Interaction}

The interaction of the Fermi gas will be described by a two-body operator $V$. It acts on the antisymmetric 2-particle space as

$$
(V \Phi)_{i_{1}, i_{2}}\left(\mathbf{x}_{1}, \mathbf{x}_{2}\right)=\sum_{i_{3}, i_{4}} \iint V_{i_{1}, i_{2}, i_{3}, i_{4}}\left(\mathbf{x}_{1}, \mathbf{x}_{2}, \mathbf{x}_{3}, \mathbf{x}_{4}\right) \Phi_{i_{4}, i_{3}}\left(\mathbf{x}_{4}, \mathbf{x}_{3}\right) \mathrm{d} \mathbf{x}_{3} \mathrm{~d} \mathbf{x}_{4}
$$

where $\Phi \in \Gamma_{\mathrm{a}}^{2}\left(L^{2}\left(\mathbb{R}^{d}, \mathbb{C}^{m}\right)\right)$. We will assume that it is self-adjoint translation invariant. Its integral kernel satisfies

$$
\begin{aligned}
V_{i_{1}, i_{2}, i_{3}, i_{4}}\left(\mathbf{x}_{1}, \mathbf{x}_{2}, \mathbf{x}_{3}, \mathbf{x}_{4}\right) & =-V_{i_{2}, i_{1}, i_{3}, i_{4}}\left(\mathbf{x}_{2}, \mathbf{x}_{1}, \mathbf{x}_{3}, \mathbf{x}_{4}\right) \\
& =-V_{i_{1}, i_{2}, i_{4}, i_{3}}\left(\mathbf{x}_{1}, \mathbf{x}_{2}, \mathbf{x}_{4}, \mathbf{x}_{3}\right) \\
& =\overline{V_{i_{4}, i_{3}, i_{2}, i_{1}}\left(\mathbf{x}_{4}, \mathbf{x}_{3}, \mathbf{x}_{2}, \mathbf{x}_{1}\right)} \\
& =V_{i_{1}, i_{2}, i_{3}, i_{4}}\left(\mathbf{x}_{1}+\mathbf{y}, \mathbf{x}_{2}+\mathbf{y}, \mathbf{x}_{3}+\mathbf{y}, \mathbf{x}_{4}+\mathbf{y}\right) .
\end{aligned}
$$

The first two identities express the antisymmetry of the interaction, the thirdits hermiticity and the fourth - its translation invariance. We also assume that $V\left(\mathbf{x}_{1}, \mathbf{x}_{2}, \mathbf{x}_{3}, \mathbf{x}_{4}\right)$ decays for large differences of its arguments sufficiently fast.

We will sometimes assume that $V$ are real, that means, they are invariant with respect to the complex conjugation. This means $V_{i_{1}, i_{2}, i_{3}, i_{4}}\left(\mathbf{x}_{1}, \mathbf{x}_{2}, \mathbf{x}_{3}, \mathbf{x}_{4}\right)$ is real.

We will say that the operator $V$ is spin independent if there exists a function $v\left(\mathbf{x}_{1}, \mathbf{x}_{2}, \mathbf{x}_{3}, \mathbf{x}_{4}\right)$ such that

$$
\begin{aligned}
& V_{i_{1}, i_{2}, i_{3}, i_{4}}\left(\mathbf{x}_{1}, \mathbf{x}_{2}, \mathbf{x}_{3}, \mathbf{x}_{4}\right) \\
& \quad=\frac{1}{2}\left(v\left(\mathbf{x}_{1}, \mathbf{x}_{2}, \mathbf{x}_{3}, \mathbf{x}_{4}\right) \delta_{i_{1}, i_{4}} \delta_{i_{2}, i_{3}}-v\left(\mathbf{x}_{1}, \mathbf{x}_{2}, \mathbf{x}_{4}, \mathbf{x}_{3}\right) \delta_{i_{1}, i_{3}} \delta_{i_{2}, i_{4}}\right),
\end{aligned}
$$


Note that

$$
\begin{aligned}
v\left(\mathbf{x}_{1}, \mathbf{x}_{2}, \mathbf{x}_{3}, \mathbf{x}_{4}\right) & =v\left(\mathbf{x}_{2}, \mathbf{x}_{1}, \mathbf{x}_{4}, \mathbf{x}_{3}\right) \\
& =\overline{v\left(\mathbf{x}_{4}, \mathbf{x}_{3}, \mathbf{x}_{2}, \mathbf{x}_{1}\right)} \\
& =v\left(\mathbf{x}_{1}+\mathbf{y}, \mathbf{x}_{2}+\mathbf{y}, \mathbf{x}_{3}+\mathbf{y}, \mathbf{x}_{4}+\mathbf{y}\right) .
\end{aligned}
$$

It will be convenient to write the Fourier transform of $V$ as follows

$$
\begin{aligned}
& V\left(\mathbf{x}_{1}, \mathbf{x}_{2}, \mathbf{x}_{3}, \mathbf{x}_{4}\right) \\
& =(2 \pi)^{-4 d} \int \mathrm{e}^{\mathrm{i} \mathbf{k}_{1} \mathbf{x}_{1}+\mathrm{i} \mathbf{k}_{2} \mathbf{x}_{2}-\mathrm{i} \mathbf{k}_{3} \mathbf{x}_{3}-\mathrm{i} \mathbf{k}_{4} \mathbf{x}_{4}} Q\left(\mathbf{k}_{1}, \mathbf{k}_{2}, \mathbf{k}_{3}, \mathbf{k}_{4}\right) \\
& \quad \times \delta\left(\mathbf{k}_{1}+\mathbf{k}_{2}-\mathbf{k}_{3}-\mathbf{k}_{4}\right) \mathrm{d} \mathbf{k}_{1} \mathrm{~d} \mathbf{k}_{2} \mathrm{~d} \mathbf{k}_{3} \mathrm{~d} \mathbf{k}_{4},
\end{aligned}
$$

where $Q\left(\mathbf{k}_{1}, \mathbf{k}_{2}, \mathbf{k}_{3}, \mathbf{k}_{4}\right)$ is a function defined on the subspace $\mathbf{k}_{1}+\mathbf{k}_{2}=\mathbf{k}_{3}+\mathbf{k}_{4}$. (Thus we could drop, say, $\mathbf{k}_{4}$ from its arguments; we do not do it for the sake of the symmetry of formulas.) Clearly,

$$
\begin{aligned}
Q_{i_{1}, i_{2}, i_{3}, i_{4}}\left(\mathbf{k}_{1}, \mathbf{k}_{2}, \mathbf{k}_{3}, \mathbf{k}_{4}\right) & =-Q_{i_{2}, i_{1}, i_{3}, i_{4}}\left(\mathbf{k}_{2}, \mathbf{k}_{1}, \mathbf{k}_{3}, \mathbf{k}_{4}\right) \\
& =-Q_{i_{1}, i_{2}, i_{4}, i_{3}}\left(\mathbf{k}_{1}, \mathbf{k}_{2}, \mathbf{k}_{4}, \mathbf{k}_{3}\right) \\
& =\overline{Q_{i_{4}, i_{3}, i_{2}, i_{1}}\left(\mathbf{k}_{4}, \mathbf{k}_{3}, \mathbf{k}_{2}, \mathbf{k}_{1}\right)}
\end{aligned}
$$

If we assume that the interaction is real, then

$$
Q_{i_{1}, i_{2}, i_{3}, i_{k}}\left(\mathbf{k}_{1}, \mathbf{k}_{2}, \mathbf{k}_{3}, \mathbf{k}_{4}\right)=\overline{Q_{i_{1}, i_{2}, i_{3}, i_{k}}\left(-\mathbf{k}_{1},-\mathbf{k}_{2},-\mathbf{k}_{3},-\mathbf{k}_{4}\right)} .
$$

If we assume that the interaction is spin-independent, then

$$
\begin{aligned}
& Q_{i_{1} i_{2} i_{3} i_{4}}\left(\mathbf{k}_{1}, \mathbf{k}_{2}, \mathbf{k}_{3}, \mathbf{k}_{4}\right) \\
& \quad=\frac{1}{2}\left(q\left(\mathbf{k}_{1}, \mathbf{k}_{2}, \mathbf{k}_{3}, \mathbf{k}_{4}\right) \delta_{i_{1} i_{4}} \delta_{i_{2} i_{3}}-q\left(\mathbf{k}_{1}, \mathbf{k}_{2}, \mathbf{k}_{4}, \mathbf{k}_{3}\right) \delta_{i_{1} i_{3}} \delta_{i_{2} i_{4}}\right),
\end{aligned}
$$

for some function $q$ defined on $\mathbf{k}_{1}+\mathbf{k}_{2}=\mathbf{k}_{3}+\mathbf{k}_{4}$ satisfying

$$
\begin{aligned}
q\left(\mathbf{k}_{1}, \mathbf{k}_{2}, \mathbf{k}_{3}, \mathbf{k}_{4}\right) & =q\left(\mathbf{k}_{2}, \mathbf{k}_{1}, \mathbf{k}_{4}, \mathbf{k}_{3}\right) \\
& =\overline{q\left(\mathbf{k}_{4}, \mathbf{k}_{3}, \mathbf{k}_{2}, \mathbf{k}_{1}\right)} .
\end{aligned}
$$

In the real spin-independent case we have in addition

$$
q\left(\mathbf{k}_{1}, \mathbf{k}_{2}, \mathbf{k}_{3}, \mathbf{k}_{4}\right)=\overline{q\left(-\mathbf{k}_{1},-\mathbf{k}_{2},-\mathbf{k}_{3},-\mathbf{k}_{4}\right)} .
$$

For example, a two-body potential $V(\mathbf{x})$ such that $V(\mathbf{x})=V(-\mathbf{x})$ corresponds to the real spin-independent interaction with

$$
\begin{aligned}
& v\left(\mathbf{x}_{1}, \mathbf{x}_{2}, \mathbf{x}_{3}, \mathbf{x}_{4}\right)=V\left(\mathbf{x}_{1}-\mathbf{x}_{2}\right) \delta\left(\mathbf{x}_{1}-\mathbf{x}_{4}\right) \delta\left(\mathbf{x}_{2}-\mathbf{x}_{3}\right) \\
& q\left(\mathbf{k}_{1}, \mathbf{k}_{2}, \mathbf{k}_{3}, \mathbf{k}_{4}\right)=\int \mathrm{d} \mathbf{q} \hat{V}(\mathbf{q}) \delta\left(\mathbf{k}_{1}-\mathbf{k}_{4}-\mathbf{q}\right) \delta\left(\mathbf{k}_{2}-\mathbf{k}_{3}+\mathbf{q}\right) .
\end{aligned}
$$




\section{3. $n$-Body Hamiltonian}

The $n$-body Hamiltonian of the homogeneous Fermi gas acts on the Hilbert space $\Gamma_{\mathrm{a}}^{n}\left(L^{2}\left(\mathbb{R}^{d}, \mathbb{C}^{m}\right)\right)$ (antisymmetric square integrable functions on $\left(\mathbb{R}^{d}\right)^{n}$ with values in $\left.\left(\mathbb{C}^{m}\right)^{\otimes n}\right)$. Let $T_{(i)}$ denote the operator $T$ acting on the $i$ th variable and $V_{(i j)}$ denote the operator $V$ acting on the $(i j)$ th pair of variables. The full $n$-body Hamiltonian equals

$$
H^{n}=\sum_{1 \leq i \leq n} T_{(i)}+\sum_{1 \leq i<j \leq n} V_{(i j)} .
$$

It commutes with the momentum operator

$$
P^{n}:=\sum_{i=1}^{n}-\mathrm{i} \nabla_{\mathbf{x}_{i}}
$$

\subsection{Putting System in a Box}

As discussed already in the previous section, to investigate homogeneous Fermi gas at positive density in thermodynamic limit it is convenient to put the system on a box $\Lambda=[-L / 2, L / 2]^{d}$ with periodic boundary conditions. This means in particular that the kinetic energy is replaced by

$$
T^{L}\left(x_{1}, x_{2}\right)=\frac{1}{L^{d}} \sum_{\mathbf{k} \in \frac{2 \pi}{L} \mathbb{Z}^{d}} \mathrm{e}^{\mathrm{ik} \cdot\left(\mathbf{x}_{1}-x_{2}\right)} \tau(\mathbf{k}),
$$

and the potential $V$ is replaced by

$$
\begin{aligned}
& V^{L}\left(\mathbf{x}_{1}, \mathbf{x}_{2}, \mathbf{x}_{3}, \mathbf{x}_{4}\right) \\
& =\frac{1}{L^{3 d}} \sum_{\substack{\mathbf{k}_{1}, \ldots, \mathbf{k}_{4} \in \frac{2 \pi}{L} \mathbb{Z}^{d}, \mathbf{k}_{1}+\mathbf{k}_{2}=\mathbf{k}_{3}+\mathbf{k}_{4}}} \mathrm{e}^{\mathrm{i} \mathbf{k}_{1} \cdot \mathbf{x}_{1}+\mathrm{i} \mathbf{k}_{2} \mathbf{x}_{2}-\mathrm{i} \mathbf{k}_{3} \mathbf{x}_{3}-\mathrm{i} \mathbf{k}_{4} \mathbf{x}_{4}} Q\left(\mathbf{k}_{1}, \mathbf{k}_{2}, \mathbf{k}_{3}, \mathbf{k}_{4}\right) .
\end{aligned}
$$

Note that $V^{L}$ is periodic with respect to the domain $\Lambda$, and $V^{L}(\mathbf{x}) \rightarrow V(\mathbf{x})$ as $L \rightarrow \infty$. The system on a torus is described by the Hamiltonian

$$
H^{L, n}=\sum_{1 \leq i \leq n} T_{(i)}^{L}+\sum_{1 \leq i<j \leq n} V_{(i j)}^{L}
$$

acting on the space $\Gamma_{\mathrm{a}}^{n}\left(L^{2}\left(\Lambda, \mathbb{C}^{m}\right)\right)$.

\subsection{Grand-Canonical Hamiltonian of the Fermi Gas}

It is convenient to put all the $n$-particle spaces into a single Fock space

$$
\Gamma_{\mathrm{a}}\left(L^{2}\left(\Lambda, \mathbb{C}^{m}\right)\right):=\bigoplus_{n=0}^{\infty} \Gamma_{\mathrm{a}}^{n}\left(L^{2}\left(\Lambda, \mathbb{C}^{m}\right)\right)
$$

with the Hamiltonian

$$
\begin{aligned}
H^{L}:= & \underset{n=0}{\infty} H^{L, n} \\
= & \int a_{\mathbf{x}, i_{1}}^{*} T_{i_{1}, i_{2}}^{L}\left(\mathbf{x}_{i_{1}}-\mathbf{x}_{i_{2}}\right) a_{\mathbf{x}, i_{2}} \mathrm{~d} \mathbf{x}_{1} \mathrm{~d} \mathbf{x}_{2} \\
& +\frac{1}{2} \iint a_{\mathbf{x}_{1}, i_{1}}^{*} a_{\mathbf{x}_{2}, i_{2}}^{*} V_{i_{1}, i_{2}, i_{3}, i_{4}}^{L}\left(\mathbf{x}_{1}, \mathbf{x}_{2}, \mathbf{x}_{3}, \mathbf{x}_{4}\right) a_{\mathbf{x}_{3}, i_{3}} a_{\mathbf{x}_{4}, i_{4}} \mathrm{~d} \mathbf{x}_{1} \mathrm{~d} \mathbf{x}_{2} \mathrm{~d} \mathbf{x}_{3} \mathrm{~d} \mathbf{x}_{4}
\end{aligned}
$$


where $a_{\mathbf{x}, i}, a_{\mathbf{x}, \mathrm{i}}^{*}$ are the usual fermionic annihilation and creation operators. The second quantized momentum and number operators are defined as

$$
\begin{aligned}
P^{L} & :=\bigoplus_{n=0}^{\infty} P^{n, L}=-\mathrm{i} \int a_{\mathbf{x}, i}^{*} \nabla_{\mathbf{x}}^{L} a_{\mathbf{x}, i} \mathrm{~d} \mathbf{x}, \\
N^{L} & :=\bigoplus_{n=0}^{\infty} n=\int a_{\mathbf{x}, i}^{*} a_{\mathbf{x}, i} \mathrm{~d} \mathbf{x} .
\end{aligned}
$$

Above we use the summation convention. In what follows we will usually omit the indices.

In the momentum representation,

$$
\begin{aligned}
H^{L}= & \sum_{\mathbf{k}} \tau(\mathbf{k}) a_{\mathbf{k}}^{*} a_{\mathbf{k}} \\
& +\frac{1}{2 L^{d}} \sum_{\mathbf{k}_{1}+\mathbf{k}_{2}=\mathbf{k}_{3}+\mathbf{k}_{4}} Q\left(\mathbf{k}_{1}, \mathbf{k}_{2}, \mathbf{k}_{3}, \mathbf{k}_{4}\right) a_{\mathbf{k}_{1}}^{*} a_{\mathbf{k}_{2}}^{*} a_{\mathbf{k}_{3}} a_{\mathbf{k}_{4}} \\
P^{L}= & \sum_{\mathbf{k}} \mathbf{k} a_{\mathbf{k}}^{*} a_{\mathbf{k}} \\
N^{L}= & \sum_{\mathbf{k}} a_{\mathbf{k}}^{*} a_{\mathbf{k}} .
\end{aligned}
$$

In the spin-independent case, the interaction equals

$$
\frac{1}{2 L^{d}} \sum_{\mathbf{k}_{1}+\mathbf{k}_{2}=\mathbf{k}_{3}+\mathbf{k}_{4}} q\left(\mathbf{k}_{1}, \mathbf{k}_{2}, \mathbf{k}_{3}, \mathbf{k}_{4}\right) a_{\mathbf{k}_{1}, i}^{*} a_{\mathbf{k}_{2}, j}^{*} a_{\mathbf{k}_{3}, j} a_{\mathbf{k}_{4}, i}
$$

In the case of a (local) potential, it is

$$
\frac{1}{2 L^{d}} \sum_{\mathbf{k}, \mathbf{k}^{\prime}, \mathbf{q}} \hat{V}(\mathbf{q}) a_{\mathbf{k}+\mathbf{q}, i}^{*} a_{\mathbf{k}^{\prime}-\mathbf{q}, j}^{*} a_{\mathbf{k}^{\prime}, j} a_{\mathbf{k}, i} .
$$

$H^{L, \pm}(\mathbf{k})$ will denote the operator $H^{L}$ restricted to the subspace $P^{L}=\mathbf{k}$, $(-1)^{N^{L}}= \pm 1$.

\subsection{Infimum of the Excitation Spectrum}

For a large class of potentials the finite volume Hamiltonians $H^{L}$ are bounded from below and have a discrete spectrum.

The ground state energy is defined as

$$
E^{L}=\inf \operatorname{sp} H^{L} \text {. }
$$

For $\mathbf{k} \in \frac{2 \pi}{L} \mathbb{Z}^{d}$ we define the infimum of the excitation spectrum in the even/odd sector in finite volume:

$$
\begin{aligned}
& \epsilon^{L,+}(\mathbf{k}):=\inf \operatorname{sp} H^{L,+}(\mathbf{k})-E^{L}, \mathbf{k} \neq 0, \\
& \epsilon^{L,+}(\mathbf{0}):=\inf \left(\operatorname{sp}\left(H^{L,+}(\mathbf{0})-E^{L}\right) \backslash\{0\}\right), \\
& \epsilon^{L,-}(\mathbf{k}):=\inf \operatorname{sp} H^{L,-}(\mathbf{k})-E^{L} .
\end{aligned}
$$

For $\mathbf{k} \in \mathbb{R}^{d}$, we would like to define the infimum of the excitation spectrum in thermodynamic limit. To this end, first we define its finite volume 
version in a "window" given by $\delta>0$ :

$$
\epsilon^{L, \delta, \pm}(\mathbf{k}):=\inf \left\{\epsilon^{L, \pm}\left(\mathbf{k}_{L}^{\prime}\right): \mathbf{k}_{L}^{\prime} \in \frac{2 \pi}{L} \mathbb{Z}^{d},\left|\mathbf{k}-\mathbf{k}_{L}^{\prime}\right|<\delta\right\} .
$$

Then we set

$$
\epsilon^{ \pm}(\mathbf{k})=\sup _{\delta>0}\left(\liminf _{L \rightarrow \infty}\left(\epsilon^{L, \delta, \pm}(\mathbf{k})\right)\right)
$$

Let us now formulate our conjectures about $\epsilon^{ \pm}$.

Conjecture 3.1. We expect that for a large class of potentials with attractive interactions the following statements hold true:

1. The functions $\mathbb{R}^{d} \ni \mathbf{k} \mapsto \epsilon^{ \pm}(\mathbf{k}) \in \mathbb{R}$ are continuous.

2. Let $\mathbf{k} \in \mathbb{R}^{d}$. Let $\left(\mathbf{k}_{s}, L_{s}\right) \in \frac{2 \pi}{L_{s}} \mathbb{Z}^{d} \times[0, \infty)$ obey $\mathbf{k}_{s} \rightarrow \mathbf{k}, L_{s} \rightarrow \infty$. Then $\epsilon^{L_{s}, \pm}\left(\mathbf{k}_{s}\right) \rightarrow \epsilon^{ \pm}(\mathbf{k})$.

3. If $d \geq 2$, then

$$
\inf _{\mathbf{k}} \min \left(\epsilon^{-}(\mathbf{k}), \epsilon^{+}(\mathbf{k})\right)=: \varepsilon>0
$$

4. If $d \geq 2$, then

$$
\inf _{\mathbf{k} \neq \mathbf{0}} \frac{\min \left(\epsilon^{-}(\mathbf{k}), \epsilon^{+}(\mathbf{k})\right)}{|\mathbf{k}|}=: c_{\mathrm{cr}}>0 .
$$

5. We have the following subadditivity properties:

$$
\begin{aligned}
& \epsilon^{-}\left(\mathbf{k}_{1}+\mathbf{k}_{2}\right) \leq \epsilon^{-}\left(\mathbf{k}_{1}\right)+\epsilon^{+}\left(\mathbf{k}_{2}\right), \\
& \epsilon^{+}\left(\mathbf{k}_{1}+\mathbf{k}_{2}\right) \leq \epsilon^{-}\left(\mathbf{k}_{1}\right)+\epsilon^{-}\left(\mathbf{k}_{2}\right), \\
& \epsilon^{+}\left(\mathbf{k}_{1}+\mathbf{k}_{2}\right) \leq \epsilon^{+}\left(\mathbf{k}_{1}\right)+\epsilon^{+}\left(\mathbf{k}_{2}\right) .
\end{aligned}
$$

To motivate the above conjecture, consider a model Hamiltonian

$$
H=\sum_{i \in \mathcal{Q}} \int_{I_{i}} \omega_{i}(\mathbf{k}) b_{\mathbf{k}, i}^{*} b_{\mathbf{k}, i} \mathrm{~d} \mathbf{k},
$$

where $b_{\mathbf{k}, i}, \quad b_{\mathbf{k}, i}^{*}$ are (fermionic, but possibly also bosonic) annihilation/ creation operators and $I_{i} \ni \mathbf{k} \mapsto \omega_{i}(\mathbf{k})$ are continuous functions defined on closed subsets $I_{i} \subset \mathbb{R}^{d}$. For $\mathbf{k} \in \mathbb{R}^{d}$, let $\omega_{\min }(\mathbf{k})$ be the lowest dispersion relation defined as in (2.14). Assume that

$$
\begin{aligned}
\varepsilon & :=\inf _{\mathbf{k}} \omega_{\min }(\mathbf{k})>0, \\
c_{\mathrm{cr}} & :=\inf _{\mathbf{k} \neq \mathbf{0}} \frac{\omega_{\min }(\mathbf{k})}{|\mathbf{k}|}>0,
\end{aligned}
$$

which is suggested by the HFB approximation, see Sect. 4. Then the infimum of the even/odd excitation spectrum of the Hamiltonian $H$ equals $\epsilon^{ \pm}(\mathbf{k})=$ $\varsigma_{\omega_{\text {min }}}^{ \pm}(\mathbf{k})$ and has the properties described in Conjecture 3.1.

Note that in this conjecture we expect statements (3) and (4) to be true only in $d \geq 2$. This is due to an argument based on the Galilean covariance in a box with periodic boundary conditions in one dimension explained in Sect. II $\mathrm{B}$ of $[8]$. It is valid both for bosons and fermions. 


\subsection{Isolated Quasiparticle Shells}

The quadratic part of the model Hamiltonian (4.11) obtained as the result of the HFB approximation involves $m$ fermionic quasiparticles (corresponding to the dimension of the "internal subspace" $\mathbb{C}^{m}$ ). Its excitation spectrum will contain "lacunas" above its infimum separated by at most $m$ shells. In this subsection we try to formulate an additional conjecture that takes these lacunas into account. This is more difficult than the conjectures from the previous subsection. It is also more dubious.

For $\mathbf{k} \in \frac{2 \pi}{L} \mathbb{Z}^{d}$ and $j, n \in \mathbb{N}$, we define the $j$ th shell in finite volume in the $n$-body case

$$
\begin{aligned}
& \nu_{j}^{L, n,+}(\mathbf{k}):=\text { the } j \text { th lowest eigenvalue of } H^{L, n,+}(\mathbf{k})-E^{L}, \mathbf{k} \neq \mathbf{0}, \\
& \nu_{j}^{L, n,+}(\mathbf{0}):=\text { the } j+1 \text { st lowest eigenvalue of } H^{L, n,+}(\mathbf{0})-E^{L}, \\
& \nu_{j}^{L, n,-}(\mathbf{k}):=\text { the } j \text { th lowest eigenvalue of } H^{L, n,-}(\mathbf{k})-E^{L} .
\end{aligned}
$$

(Of course, when counting eigenvalues we take into account their multiplicity.)

Let $\mathbb{N}^{+}:=\{0,2,4, \ldots\}$ and $\mathbb{N}^{-}:=\{1,3,5, \ldots\}$. For $\mathbf{k} \in \mathbb{R}^{d}$, we would like to define the the $j$ th shell in thermodynamic limit. To this end, first we define its finite volume version in a "window" given by $\delta>0$ :

$$
\nu_{j}^{L, \delta,+}(\mathbf{k}):=\inf _{\mathbf{k}_{L}^{\prime} \in \frac{2 \pi}{L} \mathbb{Z}^{d},\left|\mathbf{k}-\mathbf{k}_{L}^{\prime}\right|<\delta, n \in \mathbb{N}^{ \pm}}\left\{\nu_{j}^{L, n}\left(\mathbf{k}_{L}^{\prime}\right), \inf \operatorname{sp} H^{L, n}-E^{L}<\delta\right\} .
$$

Then we set

$$
\nu_{j}^{ \pm}(\mathbf{k})=\sup _{\delta>0}\left(\liminf _{L \rightarrow \infty}\left(\nu_{j}^{L, \delta, \pm}(\mathbf{k})\right)\right)
$$

Clearly,

$$
\begin{aligned}
& \nu_{1}^{ \pm}(\mathbf{k})=\epsilon^{ \pm}(\mathbf{k}), \\
& \nu_{j}^{ \pm}(\mathbf{k}) \leq \nu_{j+1}^{ \pm}(\mathbf{k}) .
\end{aligned}
$$

Set

$$
\epsilon_{\mathrm{ess}}^{ \pm}(\mathbf{k}):=\sup \left\{\nu_{j}^{ \pm}(\mathbf{k}): j=1,2, \ldots\right\} .
$$

Let us now formulate the conjectures about $\epsilon_{\mathrm{ess}}^{ \pm}$.

Conjecture 3.2. We expect that for a large class of attractive potentials the following statements hold true:

1. The functions $\mathbb{R}^{d} \ni \mathbf{k} \mapsto \nu_{j}^{ \pm}(\mathbf{k}), \epsilon_{\mathrm{ess}}^{ \pm}(\mathbf{k}) \in \mathbb{R}_{+}$are continuous.

2. Let $\left(\mathbf{k}_{s}, L_{s}, n_{s}\right) \in \frac{2 \pi}{L_{s}} \mathbb{Z}^{d} \times\left[0, \infty\left[\times \mathbb{N}^{ \pm}\right.\right.$obey $\mathbf{k}_{s} \rightarrow \mathbf{k}$, inf $\operatorname{sp} H^{L_{s}, n_{s}}-E^{L_{s}} \rightarrow$ $0, L_{s} \rightarrow \infty$. Then

$$
\nu_{j}^{L_{s}, n_{s}, \pm}\left(\mathbf{k}_{s}\right) \rightarrow \nu_{j}^{ \pm}(\mathbf{k}) .
$$

A similar property holds for $\epsilon_{\mathrm{ess}}^{ \pm}$. 
3. $\epsilon_{\mathrm{ess}}^{ \pm}$are related to $\epsilon^{ \pm}$as follows:

$$
\begin{array}{ll}
\epsilon_{\mathrm{ess}}^{-}(\mathbf{k})=\inf \left\{\epsilon^{-}\left(\mathbf{k}_{1}\right)+\epsilon^{+}\left(\mathbf{k}_{2}\right): \mathbf{k}=\mathbf{k}_{1}+\mathbf{k}_{2}\right\} \\
\epsilon_{\mathrm{ess}}^{+}(\mathbf{k})=\inf \left\{\epsilon^{+}\left(\mathbf{k}_{1}\right)+\epsilon^{+}\left(\mathbf{k}_{2}\right),\right. & \left.\epsilon^{-}\left(\mathbf{k}_{1}\right)+\epsilon^{-}\left(\mathbf{k}_{2}\right): \mathbf{k}=\mathbf{k}_{1}+\mathbf{k}_{2}\right\} .
\end{array}
$$

To justify this conjecture, let us note first that it is consistent with the spectral properties of the model Hamiltonian (3.5) if we assume that the number of quasiparticles is finite.

We can try to be more precise. We expect that the functions $\nu_{j}$ stabilize. In other words, for a certain $m^{ \pm}$and $j \geq m^{ \pm}$, all $\nu_{j}^{ \pm}$are equal to one another, and hence equal to $\epsilon_{\mathrm{ess}}^{ \pm}$. Then it is natural to guess that the functions $\omega_{j}$ that appear in the model Hamiltonian (3.5) and correspond to bosonic, resp. fermionic quasiparticles coincide with $\nu_{j}^{ \pm}$for $j \leq m^{ \pm}$.

Note that the HFB approximation, described in the next section, suggests that $m^{+}=0$ and $m^{-}=m$, where $m$ is the number of internal degrees of freedom. In particular, this would mean that all quasiparticles are fermionic. This conjecture is probably too strong. One cannot exclude that the interaction leads to a formation of quasiparticles consisting of an even number of fermions. Such quasiparticles would be of course bosonic.

\section{The Hartree-Fock-Bogoliubov Approximation Applied to Homogeneous Fermi Gas}

One can try to compute the excitation spectrum of the Fermi gas by approximate methods. Historically, the first computation of this sort is due to Bardeen-Cooper-Schrieffer.

In its original version, the BCS method involved a replacement of quadratic fermionic operators with bosonic ones. We will use the approach based on a Bogoliubov rotation of fermionic variables, which is commonly called the Hartree-Fock-Bogoliubov method. Its main idea is to minimize the energy in the so-called fermionic Gaussian states - states obtained by a Bogoliubov rotation from the fermionic Fock vacuum. The minimizing state will define new creation/annihilation operators. We express the Hamiltonian in the new creation/annihilation operators and drop all higher order terms. This defines a new Hamiltonian, that we expect to give an approximate description of low energy part of the excitation spectrum.

\subsection{The Rotated Hamiltonian}

One can start the HFB method with a rotation of the fermionic creation/ annihilation operators. For any $\mathbf{k}$ this corresponds to a substitution

$$
a_{\mathbf{k}}^{*}=c_{\mathbf{k}} b_{\mathbf{k}}^{*}+s_{\mathbf{k}} b_{-\mathbf{k}}, \quad a_{\mathbf{k}}=\bar{c}_{\mathbf{k}} b_{\mathbf{k}}+\bar{s}_{\mathbf{k}} b_{-\mathbf{k}}^{*},
$$

where $c_{\mathbf{k}}$ and $s_{\mathbf{k}}$ are matrices on $\mathbb{C}^{m}$ satisfying

$$
\begin{array}{r}
c_{\mathbf{k}} c_{\mathbf{k}}^{*}+s_{\mathbf{k}} s_{\mathbf{k}}^{*}=1, \\
c_{\mathbf{k}} s_{-\mathbf{k}}^{\#}+s_{\mathbf{k}} c_{-\mathbf{k}}^{\#}=0 .
\end{array}
$$


(* denotes the hermitian conjugation, \# denotes the transposition and ${ }^{*}$ denotes the complex conjugation).

(4.3) guarantees that $\left[a_{\mathbf{k}}^{*}, a_{-\mathbf{k}}^{*}\right]_{+}=0$. Note that $\left[a_{\mathbf{k}}^{*}, a_{\mathbf{k}^{\prime}}\right]_{+}=0$, $\left[a_{\mathbf{k}}^{*}, a_{-\mathbf{k}^{\prime}}^{*}\right]_{+}=0, \mathbf{k} \neq \mathbf{k}^{\prime}$ are satisfied automatically.

For a sequence $\frac{2 \pi}{L} \mathbb{Z}^{d} \ni \mathbf{k} \mapsto \theta_{\mathbf{k}}$ with values in matrices on $\mathbb{C}^{m}$ such that $\theta_{\mathbf{k}}=\theta_{-\mathbf{k}}$, set

$$
U_{\theta}:=\prod_{\mathbf{k}} \mathrm{e}^{-\frac{1}{2} \theta_{\mathbf{k}} a_{\mathbf{k}}^{*} a_{-\mathbf{k}}^{*}+\frac{1}{2} \theta_{\mathbf{k}}^{*} a_{\mathbf{k}} a_{-\mathbf{k}}} .
$$

It is well known that for an appropriate sequence $\theta$ we have

$$
U_{\theta}^{*} a_{\mathbf{k}} U_{\theta}=b_{\mathbf{k}}, \quad U_{\theta}^{*} a_{\mathbf{k}}^{*} U_{\theta}=b_{\mathbf{k}}^{*} .
$$

Note also that $U_{\theta}$ is the general form of an even Bogoliubov transformation commuting with $P^{L}$.

In this section we drop the superscript $L$, writing e.g., $H$ for $H^{L}$. The Hamiltonian (3.3) after the substitution (4.1) and the Wick ordering equals

$$
\begin{aligned}
H= & B \\
& +\frac{1}{2} \sum_{\mathbf{k}} O(\mathbf{k}) b_{\mathbf{k}}^{*} b_{-\mathbf{k}}^{*}+\frac{1}{2} \sum_{\mathbf{k}} \bar{O}(\mathbf{k}) b_{-\mathbf{k}} b_{\mathbf{k}}+\sum_{\mathbf{k}} D(\mathbf{k}) b_{\mathbf{k}}^{*} b_{\mathbf{k}} \\
& + \text { terms higher order in } b \text { 's. }
\end{aligned}
$$

Here are explicit formulas for $B, D(\mathbf{k})$ and $O(\mathbf{k})$ :

$$
\begin{aligned}
& B=\sum_{\mathbf{k}} \tau(\mathbf{k}) s_{\mathbf{k}} \bar{s}_{\mathbf{k}} \\
& +\frac{1}{2 L^{d}} \sum_{\mathbf{k}, \mathbf{k}^{\prime}} Q\left(\mathbf{k},-\mathbf{k},-\mathbf{k}^{\prime}, \mathbf{k}^{\prime}\right) s_{\mathbf{k}} c_{-\mathbf{k}} \bar{c}_{-\mathbf{k}^{\prime}} \bar{s}_{\mathbf{k}^{\prime}} \\
& +\frac{1}{L^{d}} \sum_{\mathbf{k}, \mathbf{k}^{\prime}} Q\left(\mathbf{k}, \mathbf{k}^{\prime}, \mathbf{k}^{\prime}, \mathbf{k}\right) s_{\mathbf{k}} s_{\mathbf{k}^{\prime}} \bar{s}_{\mathbf{k}^{\prime}} \bar{s}_{\mathbf{k}} ; \\
& O(\mathbf{k})=2 \tau(\mathbf{k}) c_{\mathbf{k}} \bar{s}_{\mathbf{k}} \\
& +\frac{1}{L^{d}} \sum_{\mathbf{k}^{\prime}} Q\left(\mathbf{k}^{\prime},-\mathbf{k}^{\prime},-\mathbf{k}, \mathbf{k}\right) s_{\mathbf{k}^{\prime}} c_{-\mathbf{k}^{\prime}} \bar{s}_{-\mathbf{k}} \bar{s}_{\mathbf{k}} \\
& +\frac{1}{L^{d}} \sum_{\mathbf{k}^{\prime}} Q\left(\mathbf{k},-\mathbf{k},-\mathbf{k}^{\prime}, \mathbf{k}^{\prime}\right) c_{\mathbf{k}} c_{-\mathbf{k}} \bar{c}_{-\mathbf{k}^{\prime}} \bar{s}_{\mathbf{k}^{\prime}} \\
& +\frac{4}{L^{d}} \sum_{\mathbf{k}^{\prime}} Q\left(\mathbf{k}, \mathbf{k}^{\prime}, \mathbf{k}^{\prime}, \mathbf{k}\right) c_{\mathbf{k}} s_{\mathbf{k}^{\prime}} \bar{s}_{\mathbf{k}^{\prime}} \bar{s}_{\mathbf{k}} \\
& D(\mathbf{k})=\tau(\mathbf{k}) c_{\mathbf{k}} \bar{c}_{\mathbf{k}}-\left(\tau(\mathbf{k}) s_{-\mathbf{k}} \bar{s}_{-\mathbf{k}}\right)^{\mathrm{T}} \\
& +\frac{1}{L^{d}} \sum_{\mathbf{k}^{\prime}} Q\left(\mathbf{k}^{\prime},-\mathbf{k}^{\prime},-\mathbf{k}, \mathbf{k}\right) s_{\mathbf{k}^{\prime}} c_{-\mathbf{k}^{\prime}} \bar{s}_{-\mathbf{k}} \bar{c}_{\mathbf{k}} \\
& +\frac{1}{L^{d}} \sum_{\mathbf{k}^{\prime}} Q\left(\mathbf{k},-\mathbf{k},-\mathbf{k}^{\prime}, \mathbf{k}^{\prime}\right) c_{\mathbf{k}} s_{-\mathbf{k}} \bar{c}_{-\mathbf{k}^{\prime}} \bar{s}_{\mathbf{k}^{\prime}}
\end{aligned}
$$




$$
\begin{aligned}
& +\frac{2}{L^{d}} \sum_{\mathbf{k}^{\prime}} Q\left(\mathbf{k}, \mathbf{k}^{\prime}, \mathbf{k}^{\prime}, \mathbf{k}\right) c_{\mathbf{k}} s_{\mathbf{k}^{\prime}} \bar{s}_{\mathbf{k}^{\prime}} \bar{c}_{\mathbf{k}} \\
& -\frac{2}{L^{d}} \sum_{\mathbf{k}^{\prime}}\left(Q\left(-\mathbf{k}, \mathbf{k}^{\prime}, \mathbf{k}^{\prime},-\mathbf{k}\right) s_{-\mathbf{k}^{\prime}} s_{\mathbf{k}^{\prime}} \bar{s}_{\mathbf{k}^{\prime}} \bar{s}_{-\mathbf{k}}\right)^{\mathrm{T}} .
\end{aligned}
$$

Note that the formulas for $B, O(\mathbf{k})$ and $D(\mathbf{k})$ are written in a special notation, whose aim is to avoid putting a big number of internal indices. The matrices $c_{\mathbf{k}}$ and $s_{\mathbf{k}}$ have two internal indices: right and left. We sum over the right internal indices, whenever we sum over the corresponding momenta. The left internal indices are contracted with the corresponding indices of $\tau$ or $Q$. The superscript $\mathrm{T}$ stands for the transposition (swapping the indices).

\subsection{Minimization Over Gaussian States}

Let $\Omega$ denote the vacuum vector. $\Omega_{\theta}:=U_{\theta}^{*} \Omega$ is the general form of an even fermionic Gaussian vector of zero momentum. Clearly,

$$
\begin{aligned}
\left(\Omega_{\theta} \mid H \Omega_{\theta}\right) & =B, \\
\left(b_{\mathbf{k}}^{*} \Omega_{\theta} \mid H b_{\mathbf{k}}^{*} \Omega_{\theta}\right) & =B+D(\mathbf{k}) .
\end{aligned}
$$

Therefore, we obtain rigorous bounds

$$
\begin{aligned}
E & \leq B, \\
E+\epsilon^{-}(\mathbf{k}) & \leq B+\inf D(\mathbf{k}) .
\end{aligned}
$$

We would like to find a fermionic Gaussian vector that minimizes $B$ - the expectation value of $H$. We assume that there exists a stationary point $\left(\tilde{s}_{\mathbf{k}}, \tilde{c}_{\mathbf{k}}\right)$ of $B$ considered as a function of $c$ and $s$. Bogoliubov transformations form a group, hence the neighborhood of the stationary point can be expressed in the following way:

$$
\left[\begin{array}{ll}
c_{\mathbf{k}} & s_{\mathbf{k}} \\
\bar{s}_{\mathbf{k}} & \bar{c}_{\mathbf{k}}
\end{array}\right]=\left[\begin{array}{ll}
\tilde{c}_{\mathbf{k}} & \tilde{s}_{\mathbf{k}} \\
\overline{\tilde{s}}_{\mathbf{k}} & \overline{\tilde{c}}_{\mathbf{k}}
\end{array}\right]\left[\begin{array}{cc}
c_{\mathbf{k}}^{\prime} & s_{\mathbf{k}}^{\prime} \\
\bar{s}_{\mathbf{k}}^{\prime} & \bar{c}_{\mathbf{k}}^{\prime}
\end{array}\right] .
$$

This means (including internal indices) that

$$
\begin{aligned}
& c_{i l, \mathbf{k}}=\tilde{c}_{i m, \mathbf{k}} c_{m l, \mathbf{k}}^{\prime}+\tilde{s}_{i m, \mathbf{k}}{\overline{s^{\prime}}}_{m l, \mathbf{k}}, \\
& \bar{c}_{i l, \mathbf{k}}=\overline{\tilde{s}}_{i m, \mathbf{k}} s_{m l, \mathbf{k}}^{\prime}+\overline{\tilde{c}}_{i m, \mathbf{k}} \bar{c}_{m l, \mathbf{k}}^{\prime}, \\
& s_{i l, \mathbf{k}}=\tilde{c}_{i m, \mathbf{k}} s_{m l, \mathbf{k}}^{\prime}+\tilde{s}_{i m, \mathbf{k}} \bar{c}_{m l, \mathbf{k}}^{\prime}, \\
& \bar{s}_{i l, \mathbf{k}}=\overline{\tilde{s}}_{i m, \mathbf{k}} c_{m l, \mathbf{k}}^{\prime}+\overline{\tilde{c}}_{i m, \mathbf{k}} \bar{s}_{m l, \mathbf{k}} .
\end{aligned}
$$

We enter the above formulas into the expressions for $B, O(\mathbf{k})$ and $D(\mathbf{k})$.

We can always multiply $c_{\mathbf{k}}$ and $s_{\mathbf{k}}$ by a unitary matrix without changing the Gaussian state. Hence, we can assume that

$$
c_{\mathbf{k}}^{\prime}=\sqrt{1-\left(s_{\mathbf{k}}^{\prime}\right) * s_{\mathbf{k}}^{\prime}} \text {. }
$$

Since $s^{\prime}$ is a complex function we can treat $s^{\prime}$ and $\bar{s}^{\prime}$ as independent variables. $c_{\mathbf{k}}=\tilde{c}_{\mathbf{k}}, s_{\mathbf{k}}=\tilde{s}_{\mathbf{k}}$ corresponds to $s^{\prime}=0, \bar{s}^{\prime}=0$. Because of (4.9), we have

$$
\left.\frac{\partial}{\partial s_{\mathbf{k}}^{\prime}} c_{\mathbf{k}}^{\prime}\right|_{\substack{s^{\prime}=0 \\ s^{\prime}=0}}=0 \text {, }
$$




$$
\left.\frac{\partial}{\partial \bar{s}_{\mathbf{k}}^{\prime}} c_{\mathbf{k}}^{\prime}\right|_{\substack{s^{\prime}=0 \\ s^{\prime}=0}}=0 .
$$

Then, for example, taking the first term of $B$ one gets

$$
\left.\frac{\partial}{\partial s_{r t, \mathbf{k}^{\prime}}^{\prime}} \sum_{\mathbf{k}} \tau_{\alpha \beta, \mathbf{k}} s_{\alpha \alpha^{\prime}, \mathbf{k}} \bar{s}_{\beta \alpha^{\prime}, \mathbf{k}}\right|_{\substack{s^{\prime}=0 \\ s^{\prime}=0}}=\tau_{\alpha \beta, \mathbf{k}^{\prime}} \tilde{c}_{\alpha r, \mathbf{k}^{\prime}} \overline{\tilde{s}}_{\beta t, \mathbf{k}^{\prime}},
$$

which equals the first term of $O(\mathbf{k})$ at $c=\tilde{c}$ and $s=\tilde{s}$. Calculating other terms of $B$ one finally gets

$$
\left.\frac{\partial B}{\partial s^{\prime}}\right|_{\substack{s^{\prime}=0 \\ s^{\prime}=0}}=\left.\frac{1}{2} O(\mathbf{k})\right|_{\substack{c=\tilde{c} \\ s=\tilde{s}}}
$$

Thus the minimizing procedure is equivalent to $O(\mathbf{k})=0$. This result is a special case of a more general fact discussed in [13] where it is called the Beliaev Theorem [2].

Thus, if we choose the Bogoliubov transformation according to the minimization procedure, the Hamiltonian equals

$$
H=B+\sum_{\mathbf{k}} D(\mathbf{k}) b_{\mathbf{k}}^{*} b_{\mathbf{k}}+\text { terms higher order in } b \text { 's. }
$$

In the case of the model interaction considered by Bardeen-CooperSchrieffer, described in many texts, e.g., in [14], the minimization of $B$ yields a dispersion relations that has a positive energy gap and a positive critical velocity uniformly as $L \rightarrow \infty$, that is,

$$
\inf _{\mathbf{k}} D(\mathbf{k})>0, \quad \inf _{\mathbf{k} \neq \mathbf{0}} \frac{D(\mathbf{k})}{|\mathbf{k}|}>0 .
$$

This phenomenon is probably much more general. In particular, we expect that it is true for a large class of real, spin-independent and attractive interactions. In what follows we provide computations that seem to support this claim.

Note that the reality and spin-independence of the interactions leads to a considerable computational simplification. By an attractive interaction we mean an interaction, which in some sense, described later on, is negative definite.

Let us assume in addition that higher order terms in (4.11) are in some sense negligible. Then formally $H$ is approximated by a quadratic Hamiltonian $B+\sum_{\mathbf{k}} D(\mathbf{k}) b_{\mathbf{k}}^{*} b_{\mathbf{k}}$ whose dispersion relation has a strictly positive energy gap and critical velocity. We view this as an argument in favor of Conjectures 3.1 and 3.2 .

\subsection{Reality Condition}

Let us first apply the assumption about the reality of the interaction. In this case, it is natural to assume that the trial vector is real as well. This means that we impose the conditions

$$
\bar{c}_{\mathbf{k}}=c_{-\mathbf{k}}, \quad \bar{s}_{\mathbf{k}}=s_{-\mathbf{k}} .
$$


This allows us to simplify the formulas for $B, D(\mathbf{k})$ and $O(\mathbf{k})$ :

$$
\begin{aligned}
B= & \sum_{\mathbf{k}} \tau(\mathbf{k}) s_{\mathbf{k}} \bar{s}_{\mathbf{k}} \\
& +\frac{1}{2 L^{d}} \sum_{\mathbf{k}, \mathbf{k}^{\prime}} Q\left(\mathbf{k},-\mathbf{k},-\mathbf{k}^{\prime}, \mathbf{k}^{\prime}\right) s_{\mathbf{k}} \bar{c}_{\mathbf{k}} c_{\mathbf{k}^{\prime}} \bar{s}_{\mathbf{k}^{\prime}} \\
& +\frac{1}{L^{d}} \sum_{\mathbf{k}, \mathbf{k}^{\prime}} Q\left(\mathbf{k}, \mathbf{k}^{\prime}, \mathbf{k}^{\prime}, \mathbf{k}\right) \bar{s}_{\mathbf{k}^{\prime}} \bar{s}_{\mathbf{k}^{\prime}} s_{\mathbf{k}^{\prime}} s_{\mathbf{k}} \\
O(\mathbf{k})= & 2 \tau(\mathbf{k}) c_{\mathbf{k}} \bar{s}_{\mathbf{k}} \\
& +\frac{1}{L^{d}} \sum_{\mathbf{k}^{\prime}} Q\left(\mathbf{k},-\mathbf{k},-\mathbf{k}^{\prime}, \mathbf{k}^{\prime}\right)\left(c_{\mathbf{k}} \bar{c}_{\mathbf{k}}-s_{\mathbf{k}} \bar{s}_{\mathbf{k}}\right) c_{\mathbf{k}^{\prime}} \bar{s}_{\mathbf{k}^{\prime}} \\
& +\frac{4}{L^{d}} \sum_{\mathbf{k}^{\prime}} Q\left(\mathbf{k}, \mathbf{k}^{\prime}, \mathbf{k}^{\prime}, \mathbf{k}\right) c_{\mathbf{k}} s_{\mathbf{k}^{\prime}} \bar{s}_{\mathbf{k}^{\prime}} \bar{s}_{\mathbf{k}}, \\
D(\mathbf{k})= & \tau(\mathbf{k})\left(c_{\mathbf{k}} \bar{c}_{\mathbf{k}}-s_{\mathbf{k}} \bar{s}_{\mathbf{k}}\right) \\
& +\frac{2}{L^{d}} \sum_{\mathbf{k}^{\prime}} Q\left(\mathbf{k},-\mathbf{k},-\mathbf{k}^{\prime}, \mathbf{k}^{\prime}\right) c_{\mathbf{k}} \bar{s}_{\mathbf{k}} c_{\mathbf{k}^{\prime}} \bar{s}_{\mathbf{k}^{\prime}} \\
& +\frac{2}{L^{d}} \sum_{\mathbf{k}^{\prime}} Q\left(\mathbf{k}, \mathbf{k}^{\prime}, \mathbf{k}^{\prime}, \mathbf{k}\right)\left(c_{\mathbf{k}} s_{\mathbf{k}^{\prime}} \bar{s}_{\mathbf{k}^{\prime}} \bar{c}_{\mathbf{k}}-s_{\mathbf{k}} s_{\mathbf{k}^{\prime}} \bar{s}_{\mathbf{k}^{\prime}} \bar{s}_{\mathbf{k}}\right) .
\end{aligned}
$$

\subsection{Spin $\frac{1}{2}$ Case}

Assume that the "spin space" is $\mathbb{C}^{2}$ and the Hamiltonian is spin independent. We make the BCS ansatz:

$$
\begin{aligned}
& c_{\mathbf{k}}=\cos \theta_{\mathbf{k}}\left[\begin{array}{cc}
1 & 0 \\
0 & 1
\end{array}\right], \\
& s_{\mathbf{k}}=\sin \theta_{\mathbf{k}}\left[\begin{array}{cc}
0 & 1 \\
-1 & 0
\end{array}\right],
\end{aligned}
$$

where, keeping in mind the reality condition, the parameters $\theta_{\mathbf{k}}$ are real. Then

$$
\begin{aligned}
B= & \sum_{\mathbf{k}} \tau(\mathbf{k})\left(1-\cos 2 \theta_{\mathbf{k}}\right) \\
& +\frac{1}{4 L^{d}} \sum_{\mathbf{k}, \mathbf{k}^{\prime}} \alpha\left(\mathbf{k}, \mathbf{k}^{\prime}\right) \sin 2 \theta_{\mathbf{k}} \sin 2 \theta_{\mathbf{k}^{\prime}} \\
& +\frac{1}{4 L^{d}} \sum_{\mathbf{k}, \mathbf{k}^{\prime}} \beta\left(\mathbf{k}, \mathbf{k}^{\prime}\right)\left(1-\cos 2 \theta_{\mathbf{k}}\right)\left(1-\cos 2 \theta_{\mathbf{k}^{\prime}}\right),
\end{aligned}
$$

where

$$
\begin{aligned}
& \alpha\left(\mathbf{k}, \mathbf{k}^{\prime}\right):=\frac{1}{2}\left(q\left(\mathbf{k},-\mathbf{k},-\mathbf{k}^{\prime}, \mathbf{k}^{\prime}\right)+q\left(-\mathbf{k}, \mathbf{k},-\mathbf{k}^{\prime}, \mathbf{k}^{\prime}\right)\right) \\
& \beta\left(\mathbf{k}, \mathbf{k}^{\prime}\right)=2 q\left(\mathbf{k}, \mathbf{k}^{\prime}, \mathbf{k}^{\prime}, \mathbf{k}\right)-q\left(\mathbf{k}^{\prime}, \mathbf{k}, \mathbf{k}^{\prime}, \mathbf{k}\right) .
\end{aligned}
$$


Note that

$$
\alpha\left(\mathbf{k}, \mathbf{k}^{\prime}\right)=\alpha\left(\mathbf{k}^{\prime}, \mathbf{k}\right), \quad \beta\left(\mathbf{k}, \mathbf{k}^{\prime}\right)=\beta\left(\mathbf{k}^{\prime}, \mathbf{k}\right) .
$$

In particular, in the case of local potentials we have

$$
\begin{aligned}
& \alpha\left(\mathbf{k}, \mathbf{k}^{\prime}\right):=\frac{1}{2}\left(\hat{V}\left(\mathbf{k}-\mathbf{k}^{\prime}\right)+\hat{V}\left(\mathbf{k}+\mathbf{k}^{\prime}\right)\right), \\
& \beta\left(\mathbf{k}, \mathbf{k}^{\prime}\right)=2 \hat{V}(\mathbf{0})-\hat{V}\left(\mathbf{k}-\mathbf{k}^{\prime}\right) .
\end{aligned}
$$

We further compute:

$$
\begin{aligned}
& O(\mathbf{k})=\left(\delta(\mathbf{k}) \cos 2 \theta_{k}+\xi(\mathbf{k}) \sin 2 \theta_{\mathbf{k}}\right)\left[\begin{array}{cc}
0 & 1 \\
-1 & 0
\end{array}\right] \\
& D(\mathbf{k})=\left(\xi(\mathbf{k}) \cos 2 \theta_{k}-\delta(\mathbf{k}) \sin 2 \theta_{k}\right)\left[\begin{array}{ll}
1 & 0 \\
0 & 1
\end{array}\right]
\end{aligned}
$$

where

$$
\begin{aligned}
& \delta(\mathbf{k})=\frac{1}{2 L^{d}} \sum_{\mathbf{k}^{\prime}} \alpha\left(\mathbf{k}, \mathbf{k}^{\prime}\right) \sin 2 \theta_{\mathbf{k}^{\prime}}, \\
& \xi(\mathbf{k})=\tau(\mathbf{k})+\frac{1}{2 L^{d}} \sum_{\mathbf{k}^{\prime}} \beta\left(\mathbf{k}, \mathbf{k}^{\prime}\right)\left(1-\cos 2 \theta_{\mathbf{k}^{\prime}}\right) .
\end{aligned}
$$

We are looking for a minimum of $B$. To this end, we first analyze critical points of $B$. We compute the derivative of $B$ :

$$
\partial_{2 \theta_{\mathbf{k}}} B=\delta(\mathbf{k}) \cos 2 \theta_{\mathbf{k}}+\xi(\mathbf{k}) \sin 2 \theta_{\mathbf{k}}
$$

The condition $\partial_{2 \theta_{\mathbf{k}}} B=0$, or equivalently $O(\mathbf{k})=0$, has many solutions. We can have

$$
\sin 2 \theta_{\mathbf{k}}=0, \quad \sin 2 \theta_{\mathbf{k}}= \pm 1,
$$

or

$$
\sin 2 \theta_{\mathbf{k}}=-\epsilon_{\mathbf{k}} \frac{\delta(\mathbf{k})}{\sqrt{\delta^{2}(\mathbf{k})+\xi^{2}(\mathbf{k})}} \neq 0, \quad \cos 2 \theta_{\mathbf{k}}=\epsilon_{\mathbf{k}} \frac{\xi(\mathbf{k})}{\sqrt{\delta^{2}(\mathbf{k})+\xi^{2}(\mathbf{k})}}
$$

where $\epsilon_{\mathbf{k}}= \pm 1$.

In particular, there are many solutions with all $\theta_{\mathbf{k}}$ satisfying (4.13). They correspond to Slater determinants and have a fixed number of particles. The solution of this kind that minimizes $B$ is called the normal or Hartree-Fock solution.

One expects that under some conditions the normal solution is not the global minimum of $B$. More precisely, one expects that a global minimum is reached by a configuration satisfying

$$
\sin 2 \theta_{\mathbf{k}}=-\frac{\delta(\mathbf{k})}{\sqrt{\delta^{2}(\mathbf{k})+\xi^{2}(\mathbf{k})}}, \quad \cos 2 \theta_{\mathbf{k}}=\frac{\xi(\mathbf{k})}{\sqrt{\delta^{2}(\mathbf{k})+\xi^{2}(\mathbf{k})}},
$$


where at least some of $\sin 2 \theta_{\mathbf{k}}$ are different from 0 . It is sometimes called a superconducting solution. In such a case we get

$$
D(\mathbf{k})=\sqrt{\xi^{2}(\mathbf{k})+\delta^{2}(\mathbf{k})}\left[\begin{array}{ll}
1 & 0 \\
0 & 1
\end{array}\right] .
$$

Thus we obtain a positive dispersion relation. One can expect that it is strictly positive, since otherwise the two functions $\delta$ and $\xi$ would have a coinciding zero, which seems unlikely. Thus we expect that the dispersion relation $D(\mathbf{k})$ has a positive energy gap.

If the interaction is small, then $\xi(\mathbf{k})$ is close to $\tau(\mathbf{k})$ and $\delta(\mathbf{k})$ is small. This implies that $D(\mathbf{k})$ is close to $|\tau(\mathbf{k})|$. If $\tau(\mathbf{k})$ has a critical velocity for large $\mathbf{k}$ and $D(\mathbf{k})$ has an energy gap, then this implies that $D(\mathbf{k})$ also has a critical velocity.

In other words, we expect that for a large class of interactions if the minimum of $B$ is reached at a superconducting state, then $D(\mathbf{k})$ satisfies (4.12).

We will not study conditions guaranteeing that a superconducting solution minimizes the energy in this paper. Let us only remark that such conditions involve some kind of negative definiteness of the quadratic form $\alpha$ - this is what we vaguely indicated by saying that the interaction is attractive. Indeed, multiply the definition of $\delta(\mathbf{k})$ with $\sin 2 \theta_{\mathbf{k}}$ and sum it up over $\mathbf{k}$. We then obtain

$$
\sum_{\mathbf{k}} \sin ^{2} 2 \theta_{\mathbf{k}} \sqrt{\delta^{2}(\mathbf{k})+\xi^{2}(\mathbf{k})}=-\frac{1}{2 L^{d}} \sum_{\mathbf{k}, \mathbf{k}^{\prime}} \sin 2 \theta_{\mathbf{k}} \alpha\left(\mathbf{k}, \mathbf{k}^{\prime}\right) \sin 2 \theta_{\mathbf{k}^{\prime}} .
$$

The left hand side of (4.17) is positive. This means that the quadratic form given by the kernel $\alpha\left(\mathbf{k}, \mathbf{k}^{\prime}\right)$ has to be negative at least at the vector given by $\sin 2 \theta_{\mathbf{k}}$.

Let us also indicate why one expects that the solution corresponding to (4.15) is a minimum of $B$. We compute the second derivative:

$$
\begin{aligned}
\partial_{2 \theta_{\mathbf{k}}} \partial_{2 \theta_{\mathbf{k}^{\prime}}} B= & \delta_{\mathbf{k}, \mathbf{k}^{\prime}}\left(-\sin 2 \theta_{\mathbf{k}} \delta(\mathbf{k})+\cos 2 \theta_{\mathbf{k}} \xi(\mathbf{k})\right) \\
& +\frac{1}{2 L^{d}} \alpha\left(\mathbf{k}, \mathbf{k}^{\prime}\right) \cos 2 \theta_{\mathbf{k}} \cos 2 \theta_{\mathbf{k}^{\prime}} \\
& +\frac{1}{2 L^{d}} \beta\left(\mathbf{k}, \mathbf{k}^{\prime}\right) \sin 2 \theta_{\mathbf{k}} \sin 2 \theta_{\mathbf{k}^{\prime}} .
\end{aligned}
$$

Substituting (4.15) to the first term on the right of (4.18) gives

$$
\delta_{\mathbf{k}, \mathbf{k}^{\prime}} \sqrt{\delta^{2}(\mathbf{k})+\xi^{2}(\mathbf{k})}
$$

which is positive definite. One can hope that the other two terms in the second derivative of $B$ do not spoil its positive definiteness.

\section{Acknowledgements}

The research of J.D. and M.N was supported in part by the National Science Center (NCN) grant No. 2011/01/B/ST1/04929. The work of M.N. was also 
supported by the Foundation for Polish Science International PhD Projects Programme co-financed by the EU European Regional Development Fund.

Open Access. This article is distributed under the terms of the Creative Commons Attribution License which permits any use, distribution, and reproduction in any medium, provided the original author(s) and the source are credited.

\section{References}

[1] Bardeen, J., Cooper, L.N., Schrieffer, J.R.: Theory of superconductivity. Phys. Rev. 108, 1175 (1957)

[2] Beliaev, S. T.: Effect of pairing correlations on nuclear properties. Mat.-Fys. Skr. Danske Vid. Selsk 31(11) (1959)

[3] Black, C.T., Ralph, D.C., Tinkham, M.: Spectroscopy of the Superconducting Gap in Individual Nanometer-Scale Aluminum Particles. Phys. Rev. Lett. 76, 688 (1996)

[4] Bogoliubov, N. N.: J. Phys. (USSR) 9, 23 (1947); J. Phys. USSR 11, 23 (1947), reprinted in D. Pines The Many-Body Problem (New York, W.A. Benjamin 1962)

[5] Bogolyubov, N.N. Jr., Brankov, J.G., Zagrebnov, V.A., Kurbatov, A.M., Tonchev, IM.S.: Some classes of exactly soluble models of problems in quantum statistical mechanics: the method of the approximating Hamiltonian. Russ. Math. Surv. 39(6), 1-50 (1984)

[6] Bratteli, O., Robinson, D.W.: Operator Algebras and Quantum Statistical Mechanics, vol. 1, 2nd edn. Springer, Berlin (1987)

[7] Bratteli, O., Robinson, D.W.: Operator Algebras and Quantum Statistical Mechanics, vol. 2, 2nd edn. Springer, Berlin (1996)

[8] Cornean, H., Dereziński, J., Ziń, P.: On the infimum of the energy-momentum spectrum of a homogeneous Bose gas. J. Math. Phys. 50, 062103 (2009)

[9] Critchley, R.H., Solomon, A.I.: A Variational Approach to Superfluidity. J. Stat. Phys. 14, 381-393 (1976)

[10] Dereziński, J.: Asymptotic completeness of long-range $N$-body quantum systems. Ann. Math. 138, 427-476 (1993)

[11] Dereziński, J.: Asymptotic completeness in quantum field theory. A class of Galilei covariant models. Rev. Math. Phys. 10, 191-233 (1998)

[12] Dereziński, J., Gérard, C.: Asymptotic completeness in quantum field theory. Massive Pauli-Fierz Hamiltonians. Rev. Math. Phys. 11, 383-450 (1999)

[13] Dereziński, J., Napiórkowski, M., Solovej, J. P.: On the minimization of Hamiltonians over pure gaussian states. Preprint, arXiv:1102.2931

[14] Fetter, A.L., Walecka, J.D.: Quantum Theory Of Many-Particle Systems. McGraw-Hill, San Francisco (1971)

[15] Fröhlich, J., Griesemer, M., Schlein, B.: Asymptotic completeness for Compton scattering. Comm. Math. Phys. 252, 415-476 (2004)

[16] Glimm, J., Jaffe, A.: Quantum Physics. A Functional Integral Point of View, 2nd edn. Springer, New York (1987)

[17] Jost, R.: The General Theory of Quantized Fields. AMS, Providence, RI (1965) 
[18] Maris, H.J.: Phonon-phonon interactions in liquid helium. Rev. Mod. Phys. 49, 341 (1977)

[19] Ring, P., Schuck, P.: The Nuclear Many-Body Problem. Springer, New York (1980)

[20] Schwarz, A. S: Mathematical foundations of quantum field theory. Nauka, Moscow (1975) (Russian)

[21] Seiringer, R.: The excitation spectrum for weakly interacting bosons. Commun. Math. Phys. 306, 565-578 (2011)

Jan Dereziński and Marcin Napiórkowski

Department of Mathematical Methods in Physics

Faculty of Physics

University of Warsaw

Hoża 74

00-682 Warsaw, Poland

e-mail: Jan.Derezinski@fuw.edu.pl;

Marcin.Napiorkowski@fuw.edu.pl

Krzysztof A. Meissner

Institute of Theoretical Physics

Faculty of Physics

University of Warsaw

Hoża 69

00-681 Warsaw, Poland

e-mail: Krzysztof.Meissner@fuw.edu.pl

Communicated by Claude Alain Pillet.

Received: October 31, 2011.

Accepted: April 18, 2012. 